Supporting Information for

\title{
Strategy for Efficient Discovery of Cocrystals via a Network-Based
}

\section{Recommendation Model}

Lulu Zheng, ${ }^{a, t}$ Bin Zhu, ${ }^{b,+}$ Zengrui $W u,{ }^{a}$ Xiaoxue Fang, ${ }^{b}$ Minghuang Hong, ${ }^{b}$ Guixia Liu, ${ }^{a}$ Weihua Li, $^{a}$ Guobin Ren ${ }^{b *}$ and Yun Tang ${ }^{a *}$

${ }^{a}$ Shanghai Key Laboratory of New Drug Design, School of Pharmacy, East China University of Science and Technology, Shanghai 200237, China

b State Key Laboratory of Bioreactor Engineering; Engineering Research Centre of Pharmaceutical Process Chemistry, Ministry of Education; Laboratory of Pharmaceutical Crystal Engineering \& Technology, School of Pharmacy, East China University of Science and Technology, Shanghai 200237, China

T These authors contributed equally to this work.

* Corresponding authors. ytang234@ecust.edu.cn (Y.T.); rgb@ecust.edu.cn (G.R.).

\section{Table of contents}

Detailed Description of Recommender Algorithms ...................................................... 2

Experimental Methods and Characterization.................................................................. 5

Parameter Searching for Parameter-Dependent Models .................................................... 7

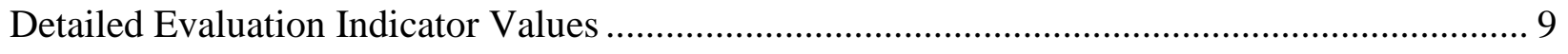

Characterization Results of Apatinib Cocrystals ............................................................ 11

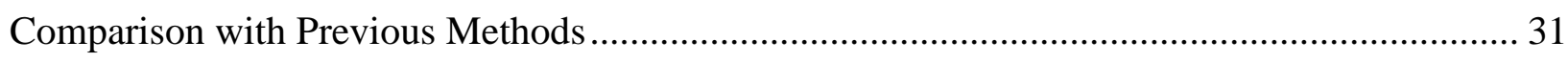

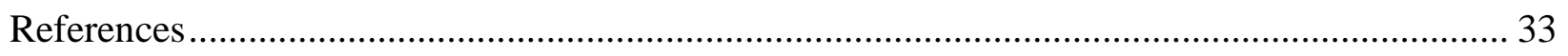




\section{Detailed Description of Recommender Algorithms}

$\mathrm{CN}$ holds that the probability that two endpoint nodes form an edge is proportion to the number of their common neighbors. Taking the influence of degree of endpoint nodes into account, Salton, Jaccard, Sørenson, HPI, HDI, LHN are presented based on CN. AA and RA are generated based on $\mathrm{CN}$ by punishing the contribution to recommendation score of common neighbors with high degrees in different ways. Given the hypothesis that the higher the number of edges connected by two endpoint nodes mediated by a certain common neighbor, the more import contribution to edge formation this common neighbor has, LNB-CN, LNB-AA and LNBRA are generated based on $\mathrm{CN}$, $\mathrm{AA}$ and $\mathrm{RA}$, respectively. $\mathrm{LP}$ is a semilocal information algorithm considering three-length paths in the calculation of recommendation scores in addition to two-length paths. LRW is random walk-based algorithm that only considering limited steps in random walk process. Katz is a global information algorithm considering all high-order paths in network. ACT, cos+ and RWR are global random walk algorithms involving global information contained in network. MFI is a global information based algorithm based on matrix-forest theory. Equations of these algorithms are provided in Tables S1 and S2. 
Table S1. Local Information Algorithms

\begin{tabular}{|c|c|c|c|}
\hline Algorithm & Formula & Algorithm & Formula \\
\hline $\mathrm{CN}^{\mathrm{a}}$ & $s_{x y}=\left|O_{x y}\right|$ & Salton ${ }^{b}$ & $s_{x y}=\frac{\left|O_{x y}\right|}{\sqrt{k_{x} k_{y}}}$ \\
\hline Jaccard $^{\mathbf{c}}$ & $s_{x y}=\frac{\left|O_{x y}\right|}{\left|U_{x y}\right|}$ & Sørenson & $s_{x y}=\frac{2\left|o_{x y}\right|}{k_{x}+k_{y}}$ \\
\hline HPI & $s_{x y}=\frac{\left|o_{x y}\right|}{\min \left\{k_{x}, k_{y}\right\}}$ & HDI & $s_{x y}=\frac{\left|o_{x y}\right|}{\max \left\{k_{x}, k_{y}\right\}}$ \\
\hline LHN & $s_{x y}=\frac{\left|o_{x y}\right|}{k_{x} k_{y}}$ & $\mathrm{AA}^{\mathbf{d}}$ & $s_{x y}=\sum_{z \in O_{x y}} \frac{1}{\log k_{z}}$ \\
\hline RA & $s_{x y}=\sum_{z \in O_{x y}} \frac{1}{k_{z}}$ & $\mathrm{LNB}^{-C N}{ }^{\mathrm{e}}$ & $s_{x y}=\left|O_{x y}\right| \log s+\sum_{z \in O_{x y}} \log R_{z}$ \\
\hline LNB-AA & $s_{x y}=\sum_{z \in O_{x y}} \frac{1}{\log k_{z}}\left(\log s+\log R_{z}\right)$ & LNB-RA & $s_{x y}=\sum_{z \in O_{x y}} \frac{1}{k_{z}}\left(\log s+\log R_{z}\right)$ \\
\hline
\end{tabular}

${ }^{\mathrm{a}} O_{x y}$ is the intersection of neighbors of node $x$ and $y$, thus $\left|O_{x y}\right|$ denotes the number of common neighbors shared by $x$ and $y$.

${ }^{\mathbf{b}} k$ is the degree of node in network.

c $\left|U_{x y}\right|$ stands for the number of union set of neighbors possessed by node $x$ and $y$.

${ }^{\mathbf{d}} z$ denotes the common neighbor of $x$ and $y$, and $k_{z}$ denotes its degree.

${ }^{\mathrm{e}} R_{Z}$ is the contribution function of $z$ defined as $R_{Z}=\frac{N_{\Delta}+1}{N_{\Lambda}+1}$, where $N_{\Delta}$ and $N_{\Lambda}$ stands for the numbers of connected and unconnected node pairs mediated by $z$, respectively. $s$ is a constant for a given network and training set defined as $\mathrm{s}=\frac{|U|}{\left|E^{\text {train }}\right|}-1$, in which $|U|$ and $\left|E^{\text {train }}\right|$ are the number of max possible edges in network and edges in $E^{\text {train }}$, respectively. 
Table S2. Semilocal and Global Information Algorithms

\begin{tabular}{|c|c|c|c|}
\hline Algorithm & Formula & Algorithm & Formula \\
\hline \multicolumn{4}{|c|}{ Semilocal information algorithms } \\
\hline $\mathrm{LP}^{\mathbf{a}}$ & $s_{x y}=\left[A^{2}\right]_{x y}+\alpha\left[A^{3}\right]_{x y}$ & $\mathrm{LRW}^{\mathbf{b}}$ & $s_{x y}(t)=\frac{k_{x}}{2 M} \pi_{x y}(t)+\frac{k_{y}}{2 M} \pi_{y x}(t)$ \\
\hline \multicolumn{4}{|c|}{ Global information algorithms } \\
\hline $\mathrm{Katz}^{\mathbf{c}}$ & $s_{x y}=\sum_{l=1}^{\infty} \beta^{l}\left|p a t h s_{x, y}^{\langle l\rangle}\right|$ & $\mathrm{ACT}^{\mathbf{d}}$ & $s_{x y}=\frac{1}{l_{x x}^{+}+l_{y y}^{+}-2 l_{x y}^{+}}$ \\
\hline $\cos +$ & $s_{x y}=\frac{l_{x y}^{+}}{\sqrt{l_{x x}^{+} \cdot l_{y y}^{+}}}$ & $\mathrm{RWR}^{\mathrm{e}}$ & $s_{x y}=\pi_{x y}+\pi_{y x}$ \\
\hline $\mathrm{MFI}^{\mathbf{f}}$ & $\boldsymbol{S}=(\boldsymbol{I}+\boldsymbol{L})^{-1}$ & & \\
\hline
\end{tabular}

${ }^{\mathrm{a}}\left[\boldsymbol{A}^{2}\right]_{x y}$ and $\left[\boldsymbol{A}^{3}\right]_{x y}$ are the number two- and three-length paths liking $x$ and $y . \alpha$, an adjustable parameter, controls the contribution of three-length paths.

${ }^{\mathbf{b}} \boldsymbol{\pi}_{x}(t)=\boldsymbol{P}^{\mathrm{T}} \boldsymbol{\pi}_{x}(t-1)$, where $\boldsymbol{P}$ is the Markov probability transfer matrix, thus $\pi_{x y}(t)$ is the probability that a random walker starting from node $x$ locates at $y$ after $t$ steps.

c $\left|p a t h s_{x, y}^{\langle l\rangle}\right|$ denotes the number of $l$-length paths linking $x$ and $y . \beta$ is an adjustable parameter that controls the weight of different paths in network.

${ }^{\text {d }}$ Defined $\boldsymbol{L}^{+}$the pseudo-inverse of laplacian matrix of network, $l_{x y}^{+}$is the item locating at row $x$ and column $y$ in $\boldsymbol{L}^{+}$.

${ }^{\mathbf{e}} \boldsymbol{\pi}_{x}=(1-c)\left(\boldsymbol{I}-c \boldsymbol{P}^{\mathrm{T}}\right)^{-1} \boldsymbol{e}^{x}$, where $(1-c), \boldsymbol{I}, \boldsymbol{P}$ and $\boldsymbol{e}^{x}$ denote return probability, unit matrix, Markov probability transfer matrix and original state, respectively. $\boldsymbol{\pi}_{x y}$ is the probability that a random walker starting from node $x$ eventually locates at $y$.

${ }^{\mathrm{f}} \boldsymbol{S}$ is the matrix form of recommendation scores. 


\section{Experimental Methods and Characterization}

\section{Cocrystallization Screening.}

The suspensions of corresponding molar amounts of APA and coformers were stirred in EtOH or EA at room temperature for 24 hours. Solid products were recovered by centrifugation, air-dried and analyzed by powder X-ray diffraction (PXRD) as dry powders. The results are summarized in Table S5.

The suspensions of corresponding molar amounts of APA and coformers were stirred in EtOH or EA at $700 \mathrm{rpm}$ and heated to $50{ }^{\circ} \mathrm{C}$, equilibrated for 4 hours until clarification. Then the clear solutions were cooled to $10{ }^{\circ} \mathrm{C}$. The results of the cooling crystallization screen are summarized in Table S6.

Suspensions/solutions of corresponding molar amounts of APA and coformers were stirred in $\mathrm{EtOH}$ or EA at $700 \mathrm{rpm}$ and heated to $50{ }^{\circ} \mathrm{C}$, equilibrated for 4 hours, then filtered through $0.45 \mu \mathrm{m}$ syringe filters into clean vials. The filtrate was slowly evaporated at RT. Residues after either evaporation were dried and analyzed by PXRD and differential scanning calorimetry (DSC). The evaporative crystallization results are summarized in Table S7.

\section{Characterization}

Single-crystal X-ray diffraction data for crystals were collected on a Bruker APEX-II CCD using $\mathrm{Cu}-\mathrm{Ka}$ radiation $(\lambda=1.54178 \AA)$ at $296 \mathrm{~K}$. Indexing was performed using APEX3 software. ${ }^{1}$ Data integration and reduction were performed using SAINT V8.38A program. ${ }^{2}$ Absorption correction was performed by a multiscan method implemented in SADABS. ${ }^{3}$ Space groups were determined using XPREP ${ }^{4}$ implemented in APEX3. The structures were solved by direct methods using ShelXT ${ }^{5}$ and were refined on $F^{2}$ using the full-matrix least-squares technique of SHELXL-2014 program packages $^{6}$ in the Olex 2 software. ${ }^{7}$ All non-hydrogen atoms 
were refined anisotropically. Hydrogen atoms were treated by a mixture of independent and constrained refinement.

PXRD patterns of solid materials were collected at ambient temperature on a Rigaku Ultima IV diffractometer (Japan) with $\mathrm{Cu}-\mathrm{K} \alpha$ radiation $(\lambda=1.541 \AA$, $40 \mathrm{kV}, 40 \mathrm{~mA})$. The scanning $2 \theta$ range was of $3-45^{\circ}$ with scanning rate of $8^{\circ} \cdot \mathrm{min}^{-1}$. The data were analyzed with Jade 6.0 .

DSC was carried out on a TA Q2000 instrument. The samples were heated from 30 or 40 to $250{ }^{\circ} \mathrm{C}$ at a heating rate of $10{ }^{\circ} \mathrm{C} \cdot \mathrm{min}^{-1}$ under nitrogen purge gas. The instruments were calibrated according to the standard procedure.

Fourier-transform infrared (FT-IR) spectra were collected by an Agilent Cary 630 FT-IR spectrometer. Sample was grinded with $\mathrm{KBr}$ (mass ratio of 200:1) into homogenous mixture using an agate mortar. Then the mixture was pressed into a pellet under vacuum condition with a compensation force of 2 tons and a $7 \mathrm{~mm}$ diameter round flat force punch.

Thermogravimetric analysis (TGA) was carried out on a TA Q500 instrument. The samples were heated from $30{ }^{\circ} \mathrm{C}$ to the decomposition temperature at a heating rate of $10{ }^{\circ} \mathrm{C} \cdot \mathrm{min}-1 \mathrm{under}$ nitrogen purge gas. 


\section{Parameter Searching for Parameter-Dependent Models}
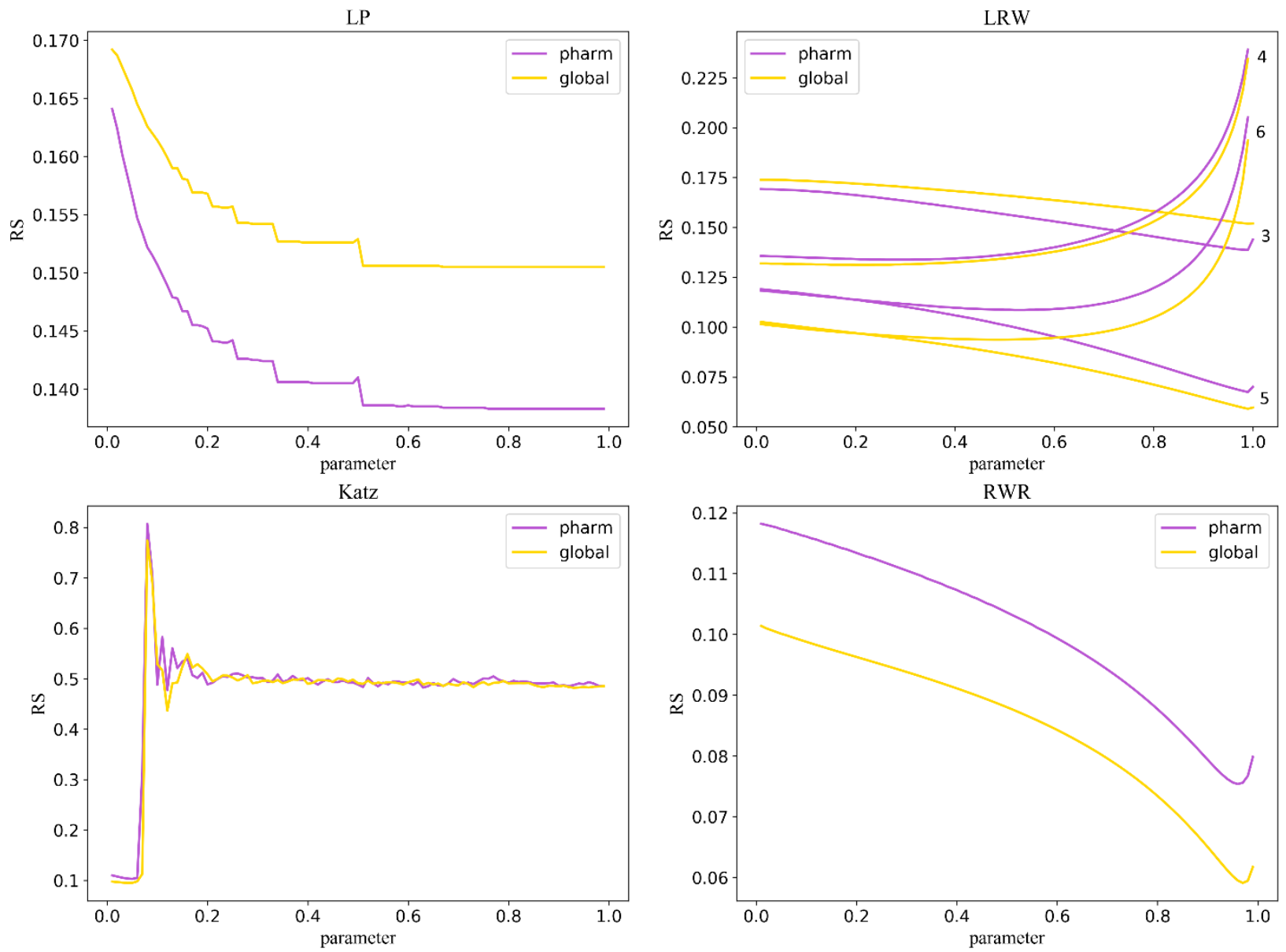

Figure S1. The relationships among parameters and $R S$ values for LP, LRW, Katz and RWR models in the whole cocrystal network and pharmaceutical cocrystal subset. The step of random walk in LRW are labeled in the plot. The performance of step 5 approaching the average shortest distance overmatched the steps of 3,4 and 6. Each parameter searching was implemented with 100 independently random runs, and corresponding $R S$ was generated by averaging these 100 independent calculations. 
Table S3. Optimal Parameters of Parameter-Dependent Models

\begin{tabular}{ccccc}
\hline Algorithm & LP & LRW-5 & Katz & RWR \\
\hline The whole network & 0.67 & 0.99 & 0.04 & 0.97 \\
Pharmaceutical cocrystal subset & 0.76 & 0.99 & 0.05 & 0.96 \\
\hline
\end{tabular}




\section{Detailed Evaluation Indicator Values}
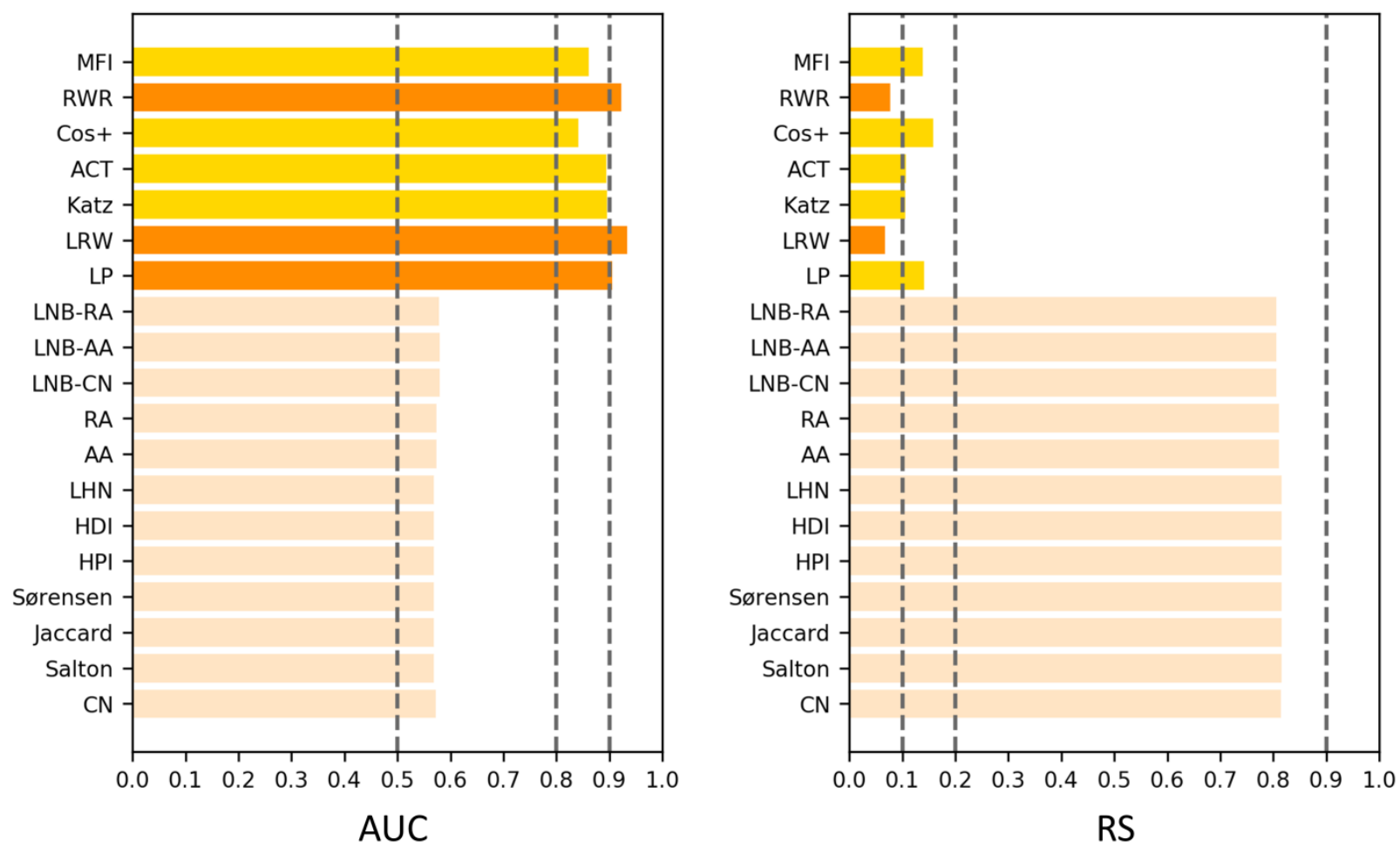

Figure S2. The comparison diagram of $A U C$ and $R S$ for recommendation models implemented in the pharmaceutical cocrystal subset. Detailed numerical values were provided in Table S5 
Table S4. $A U C$ and $R S$ Values of Recommendation Models ${ }^{\text {a }}$

\begin{tabular}{|c|c|c|c|c|}
\hline & \multicolumn{2}{|c|}{ The whole network } & \multicolumn{2}{|c|}{ Pharmaceutical cocrystal subset } \\
\hline & AUC & $\mathrm{RS}$ & AUC & $\mathrm{RS}$ \\
\hline $\mathrm{CN}$ & $0.5493 \pm 0.0094$ & $0.8724 \pm 0.0183$ & $0.5722 \pm 0.0167$ & $0.8149 \pm 0.0306$ \\
\hline Salton & $0.5472 \pm 0.0095$ & $0.8729 \pm 0.0183$ & $0.5685 \pm 0.0157$ & $0.8160 \pm 0.0305$ \\
\hline Jaccard & $0.5473 \pm 0.0098$ & $0.8727 \pm 0.0183$ & $0.5692 \pm 0.0157$ & $0.8155 \pm 0.0306$ \\
\hline Sørensen & $0.5473 \pm 0.0094$ & $0.8727 \pm 0.0183$ & $0.5690 \pm 0.0156$ & $0.8155 \pm 0.0306$ \\
\hline HPI & $0.5475 \pm 0.0095$ & $0.8730 \pm 0.0183$ & $0.5685 \pm 0.0156$ & $0.8161 \pm 0.0305$ \\
\hline HDI & $0.5477 \pm 0.0091$ & $0.8726 \pm 0.0183$ & $0.5689 \pm 0.0155$ & $0.8153 \pm 0.0307$ \\
\hline $\mathrm{LHN}$ & $0.5472 \pm 0.0092$ & $0.8730 \pm 0.0183$ & $0.5681 \pm 0.0154$ & $0.8162 \pm 0.0306$ \\
\hline AA & $0.5495 \pm 0.0096$ & $0.8705 \pm 0.0186$ & $0.5732 \pm 0.0164$ & $0.8113 \pm 0.0313$ \\
\hline RA & $0.5493 \pm 0.0095$ & $0.8705 \pm 0.0186$ & $0.5732 \pm 0.0162$ & $0.8113 \pm 0.0313$ \\
\hline LNB-CN & $0.5553 \pm 0.0105$ & $0.8651 \pm 0.0190$ & $0.5793 \pm 0.0169$ & $0.8052 \pm 0.0311$ \\
\hline LNB-AA & $0.5551 \pm 0.0107$ & $0.8652 \pm 0.0190$ & $0.5793 \pm 0.0166$ & $0.8053 \pm 0.0311$ \\
\hline LNB-RA & $0.5549 \pm 0.0103$ & $0.8654 \pm 0.0190$ & $0.5790 \pm 0.0169$ & $0.8055 \pm 0.0311$ \\
\hline LP & $0.9071 \pm 0.0117$ & $0.1489 \pm 0.0195$ & $0.9057 \pm 0.0168$ & $0.1406 \pm 0.0264$ \\
\hline LRW & $0.9439 \pm 0.0075$ & $0.0572 \pm 0.0072$ & $0.9332 \pm 0.0117$ & $0.0677 \pm 0.0121$ \\
\hline Katz & $0.9065 \pm 0.0100$ & $0.0933 \pm 0.0094$ & $0.8952 \pm 0.0149$ & $0.1052 \pm 0.0148$ \\
\hline $\mathrm{ACT}$ & $0.9054 \pm 0.0110$ & $0.0946 \pm 0.0106$ & $0.8941 \pm 0.0162$ & $0.1062 \pm 0.0154$ \\
\hline Cost & $0.8527 \pm 0.0132$ & $0.1469 \pm 0.0134$ & $0.8417 \pm 0.0203$ & $0.1587 \pm 0.0198$ \\
\hline RWR & $0.9420 \pm 0.0059$ & $0.0581 \pm 0.0058$ & $0.9231 \pm 0.0116$ & $0.0771 \pm 0.0110$ \\
\hline MFI & $0.8802 \pm 0.0098$ & $0.1202 \pm 0.0093$ & $0.8612 \pm 0.0136$ & $0.1383 \pm 0.0133$ \\
\hline
\end{tabular}

${ }^{a}$ Values of evaluation indicators are expressed as mean \pm SD in 100 independent runs. 


\section{Characterization Results of Apatinib Cocrystals}

Table S5. Summary of Slurry Screening Experiments ${ }^{a}$

\begin{tabular}{ccccc}
\hline Rank & Coformers & Solvents & Molar ratios & Forms \\
\hline 1 & MA & EA & $1: 1$ & NEW \\
2 & SUA & EtOH & $2: 1$ & NEW \\
3 & FMA & EtOH & $2: 1$ & NEW \\
4 & SBA & EtOH & $2: 1$ & NEW+CCF \\
5 & PHBA & EtOH & $1: 1$ & NEW \\
6 & GA & EtOH & $2: 1$ & NEW \\
7 & AA & EtOH & $2: 1$ & NEW \\
8 & BA & EtOH & $1: 1$ & NEW \\
9 & PA & EtOH & $2: 1$ & NEW \\
10 & DDA & EtOH & $2: 1$ & APA \\
11 & SA & EtOH & $1: 1$ & NEW \\
12 & MAA & EtOH & $2: 1$ & NEW \\
13 & OA & EtOH & $2: 1$ & NEW \\
14 & PABA & EtOH & $1: 1$ & NEW+CCF \\
15 & MHBA & EtOH & $1: 1$ & APA \\
16 & DHBA & EtOH & $1: 1$ & NEW+CCF \\
17 & DMSA & EtOH & $2: 1$ & NEW \\
$18-1$ & MSA & EtOH & $2: 1$ & APA \\
$18-2$ & MSA & EA & $2: 1$ & NEW \\
20 & OGA & EA & $2: 1$ & NEW \\
21 & HMBA & EtOH & $1: 1$ & NEW+CCF \\
\hline
\end{tabular}

${ }^{a}$ Molar ratios represented the corresponding molar ratios between APA and coformers. NEW represented PXRD of solid products are different form APA and coformers. CCF represented the corresponding cocrystal coformer. 
Table S6. Summary of Cooling Crystallization Experiments

\begin{tabular}{|c|c|c|c|c|}
\hline Rank & Conformers & Solvents & Molar ratios & Forms \\
\hline 1 & MA & EA & $1: 1$ & APA \\
\hline 2 & SUA & $\mathrm{EtOH}$ & $2: 1$ & APA \\
\hline 3 & FMA & $\mathrm{EtOH}$ & $2: 1$ & NEW \\
\hline 4 & SBA & $\mathrm{EtOH}$ & $2: 1$ & NEW \\
\hline 5 & PHBA & $\mathrm{EtOH}$ & $1: 1$ & APA \\
\hline 6 & GA & $\mathrm{EtOH}$ & $2: 1$ & APA \\
\hline 7 & AA & $\mathrm{EtOH}$ & $2: 1$ & APA \\
\hline 8 & BA & $\mathrm{EtOH}$ & $1: 1$ & APA \\
\hline 9 & PA & $\mathrm{EtOH}$ & $2: 1$ & N.A. \\
\hline 10 & DDA & $\mathrm{EtOH}$ & $2: 1$ & APA \\
\hline 11 & SA & $\mathrm{EtOH}$ & $1: 1$ & NEW \\
\hline 12 & MAA & $\mathrm{EtOH}$ & $2: 1$ & NEW \\
\hline 13 & OA & $\mathrm{EtOH}$ & $2: 1$ & N.A. \\
\hline 14 & PABA & $\mathrm{EtOH}$ & $1: 1$ & APA \\
\hline 15 & MHBA & $\mathrm{EtOH}$ & $1: 1$ & APA \\
\hline 16 & DHBA & $\mathrm{EtOH}$ & $1: 1$ & N.A. \\
\hline 17 & DMSA & $\mathrm{EtOH}$ & $2: 1$ & APA \\
\hline 18 & MSA & $\mathrm{EtOH}$ & $2: 1$ & APA \\
\hline 20 & OGA & EA & $2: 1$ & N.A. \\
\hline 21 & HMBA & $\mathrm{EtOH}$ & $1: 1$ & APA \\
\hline
\end{tabular}

${ }^{\mathrm{a}}$ N.A. represented not available. 
Table S7. Summary of Evaporative Crystallization Experiments

\begin{tabular}{|c|c|c|c|c|}
\hline Rank & Conformers & Solvents & Molar ratios & Forms \\
\hline 1 & MA & EA & $1: 1$ & NEW \\
\hline 2 & SUA & $\mathrm{EtOH}$ & $2: 1$ & NEW \\
\hline 3 & FMA & $\mathrm{EtOH}$ & $2: 1$ & NEW \\
\hline 4 & SBA & $\mathrm{EtOH}$ & $2: 1$ & NEW \\
\hline 5 & PHBA & $\mathrm{EtOH}$ & $1: 1$ & NEW \\
\hline 6 & GA & $\mathrm{EtOH}$ & $2: 1$ & NEW \\
\hline 7 & AA & $\mathrm{EtOH}$ & $2: 1$ & NEW \\
\hline 8 & BA & $\mathrm{EtOH}$ & $1: 1$ & NEW \\
\hline 9 & PA & $\mathrm{EtOH}$ & $2: 1$ & $\mathrm{NEW}+\mathrm{APA}$ \\
\hline 10 & DDA & $\mathrm{EtOH}$ & $2: 1$ & NEW \\
\hline 11 & SA & $\mathrm{EtOH}$ & $1: 1$ & NEW \\
\hline 12 & MAA & EtOH & $2: 1$ & N.A. \\
\hline 13 & $\mathrm{OA}$ & $\mathrm{EtOH}$ & $2: 1$ & N.A. \\
\hline 14 & PABA & $\mathrm{EtOH}$ & $1: 1$ & NEW \\
\hline 15 & MHBA & $\mathrm{EtOH}$ & $1: 1$ & NEW \\
\hline 16 & DHBA & $\mathrm{EtOH}$ & $1: 1$ & NEW \\
\hline 17 & DMSA & $\mathrm{EtOH}$ & $2: 1$ & APA \\
\hline $18-1$ & MSA & $\mathrm{EtOH}$ & $2: 1$ & APA \\
\hline $18-2$ & MSA & EA & $2: 1$ & NEW \\
\hline 20 & OGA & EA & $2: 1$ & APA \\
\hline 21 & HMBA & $\mathrm{EtOH}$ & $1: 1$ & $\mathrm{CCF}$ \\
\hline
\end{tabular}




\section{Preparation of the Predicted Cocrystal: Best Recipes}

APA-MA: APA $(7.95 \mathrm{mg})$ and MA $(3.04 \mathrm{mg})$ were stirred in $1 \mathrm{~mL}$ EA at $700 \mathrm{rpm}$ and heated to $50{ }^{\circ} \mathrm{C}$, equilibrated for 4 hours, then filtered through $0.45 \mu \mathrm{m}$ syringe filters into clean vials. The filtrate was slowly evaporated at RT. Solid products were air-dried and analyzed by PXRD and DSC.

APA-SUA: The suspensions of APA $(16.00 \mathrm{mg})$ and SUA $(2.36 \mathrm{mg})$ were stirred in $1 \mathrm{~mL} \mathrm{EtOH}$ at room temperature for 24 hours. Solid products were recovered by centrifugation, air-dried and analyzed by PXRD and DSC.

APA-FMA: The suspensions of APA (11.91 mg) and FMA $(1.74 \mathrm{mg})$ were stirred in 1mL EtOH at $700 \mathrm{rpm}$ and heated to $50{ }^{\circ} \mathrm{C}$ for 4 hours until clarification. Then the clear solutions were cooled to $10{ }^{\circ} \mathrm{C}$ at rate of $1{ }^{\circ} \mathrm{C} \cdot \mathrm{min}^{-1}$. Solid products were recovered by vacuum filtration, airdried and analyzed by PXRD and DSC.

APA-SBA: The suspensions of APA $(11.91 \mathrm{mg})$ and SBA $(2.61 \mathrm{mg})$ were stirred in $1 \mathrm{~mL} \mathrm{EtOH}$ at $700 \mathrm{rpm}$ and heated to $50{ }^{\circ} \mathrm{C}$ for 4 hours until clarification. Then the clear solutions were cooled to $10{ }^{\circ} \mathrm{C}$ at rate of $1{ }^{\circ} \mathrm{C} \cdot \mathrm{min}^{-1}$. Solid products were recovered by vacuum filtration, airdried and analyzed by PXRD and DSC.

APA-PHBA: The suspensions of APA (16.00 mg) and PHBA (5.52 mg) were stirred in $1 \mathrm{~mL}$ $\mathrm{EtOH}$ at room temperature for 24 hours. Solid products were recovered by centrifugation, airdried and analyzed by PXRD and DSC.

APA-GA: APA $(7.95 \mathrm{mg})$ and GA $(1.32 \mathrm{mg})$ were stirred in $1 \mathrm{~mL}$ EtOH at $700 \mathrm{rpm}$ and heated to $50{ }^{\circ} \mathrm{C}$, equilibrated for 4 hours, then filtered through $0.45 \mu \mathrm{m}$ syringe filters into clean vials. The filtrate was slowly evaporated at RT. Solid products were air-dried and analyzed by PXRD and DSC. 
APA-AA: APA $(7.95 \mathrm{mg})$ and AA $(1.88 \mathrm{mg})$ were stirred in $1 \mathrm{~mL} \mathrm{EtOH}$ at $700 \mathrm{rpm}$ and heated to $50{ }^{\circ} \mathrm{C}$, equilibrated for 4 hours, then filtered through $0.45 \mu \mathrm{m}$ syringe filters into clean vials. The filtrate was slowly evaporated at RT. Solid products were air-dried and analyzed by PXRD and DSC.

APA-BA: The suspensions of APA (16.00 mg) and BA (4.88 mg) were stirred in 1mL EtOH at room temperature for 24 hours. Solid products were recovered by centrifugation, air-dried and analyzed by PXRD and DSC.

APA-PA: The suspensions of APA (16.00 mg) and PA $(3.20 \mathrm{mg})$ were stirred in $1 \mathrm{~mL}$ EtOH at room temperature for 24 hours. Solid products were recovered by centrifugation, air-dried and analyzed by PXRD and DSC.

APA-DDA: APA $(7.95 \mathrm{mg})$ and DDA $(2.30 \mathrm{mg})$ were stirred in $1 \mathrm{~mL}$ EA at $700 \mathrm{rpm}$ and heated to $50{ }^{\circ} \mathrm{C}$, equilibrated for 4 hours, then filtered through $0.45 \mu \mathrm{m}$ syringe filters into clean vials. The filtrate was slowly evaporated at RT. Solid products were air-dried and analyzed by PXRD and DSC.

APA-SA: APA $(7.95 \mathrm{mg})$ and SA $(2.76 \mathrm{mg})$ were stirred in $1 \mathrm{~mL}$ EtOH at $700 \mathrm{rpm}$ and heated to $50{ }^{\circ} \mathrm{C}$, equilibrated for 4 hours, then filtered through $0.45 \mu \mathrm{m}$ syringe filters into clean vials. The filtrate was slowly evaporated at RT. Solid products were air-dried and analyzed by PXRD and DSC.

APA-MAA: The suspensions of APA (16.00 mg) and MAA (2.08 mg) were stirred in $1 \mathrm{~mL}$ EtOH at room temperature for 24 hours. Solid products were recovered by centrifugation, airdried and analyzed by PXRD and DSC.

APA-OA: The suspensions of APA (11.91 mg) and OA $(1.89 \mathrm{mg})$ were stirred in $1 \mathrm{~mL}$ EtOH at $700 \mathrm{rpm}$ and heated to $50{ }^{\circ} \mathrm{C}$ for 4 hours until clarification. Then the clear solutions were cooled 
to $10{ }^{\circ} \mathrm{C}$ at rate of $1{ }^{\circ} \mathrm{C} \cdot \mathrm{min}^{-1}$. Solid products were recovered by vacuum filtration, air-dried and analyzed by PXRD and DSC.

APA-PABA: APA $(7.95 \mathrm{mg})$ and PABA $(2.74 \mathrm{mg})$ were stirred in $1 \mathrm{~mL}$ EtOH at $700 \mathrm{rpm}$ and heated to $50{ }^{\circ} \mathrm{C}$, equilibrated for 4 hours, then filtered through $0.45 \mu \mathrm{m}$ syringe filters into clean vials. The filtrate was slowly evaporated at RT. Solid products were air-dried and analyzed by PXRD and DSC.

APA-MHBA: APA (7.95 mg) and MHBA $(2.76 \mathrm{mg})$ were stirred in $1 \mathrm{~mL}$ EtOH at $700 \mathrm{rpm}$ and heated to $50{ }^{\circ} \mathrm{C}$, equilibrated for 4 hours, then filtered through $0.45 \mu \mathrm{m}$ syringe filters into clean vials. The filtrate was slowly evaporated at RT. Solid products were air-dried and analyzed by PXRD and DSC.

APA-DHBA: APA $(7.95 \mathrm{mg})$ and DHBA $(3.08 \mathrm{mg})$ were stirred in $1 \mathrm{~mL} \mathrm{EtOH}$ at $700 \mathrm{rpm}$ and heated to $50{ }^{\circ} \mathrm{C}$, equilibrated for 4 hours, then filtered through $0.45 \mu \mathrm{m}$ syringe filters into clean vials. The filtrate was slowly evaporated at RT. Solid products were air-dried and analyzed by PXRD and DSC.

APA-DMSA: The suspensions of APA (16.00 mg) and DMSA $(2.92 \mathrm{mg})$ were stirred in $1 \mathrm{~mL}$ $\mathrm{EtOH}$ at room temperature for 24 hours. Solid products were recovered by centrifugation, airdried and analyzed by PXRD and DSC.

APA-MSA: APA $(7.95 \mathrm{mg})$ and MSA $(1.32 \mathrm{mg})$ were stirred in $1 \mathrm{~mL}$ EA at $700 \mathrm{rpm}$ and heated to $50{ }^{\circ} \mathrm{C}$, equilibrated for 4 hours, then filtered through $0.45 \mu \mathrm{m}$ syringe filters into clean vials. The filtrate was slowly evaporated at RT. Solid products were air-dried and analyzed by PXRD and DSC.

APA-OGA: The suspensions of APA (16.00 mg) and OGA (2.92 mg) were stirred in 1mL EtOH at room temperature for 24 hours. Solid products were recovered by centrifugation, air-dried and 
analyzed by PXRD and DSC. 


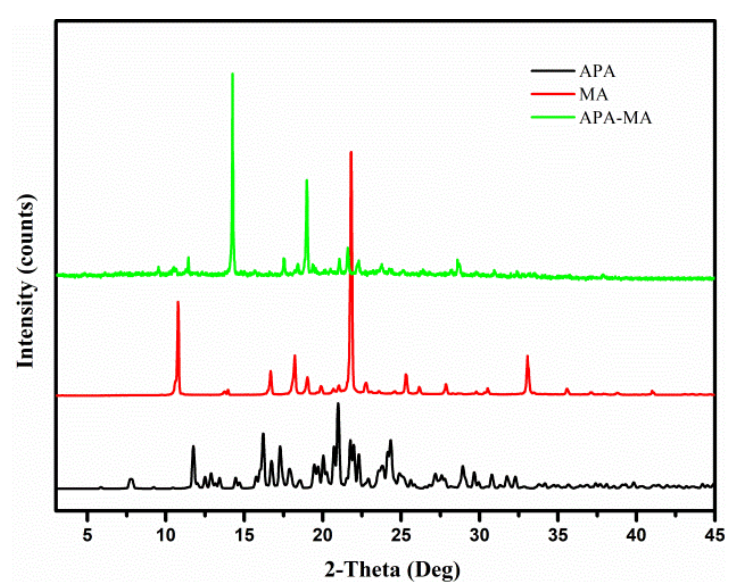

APA-MA

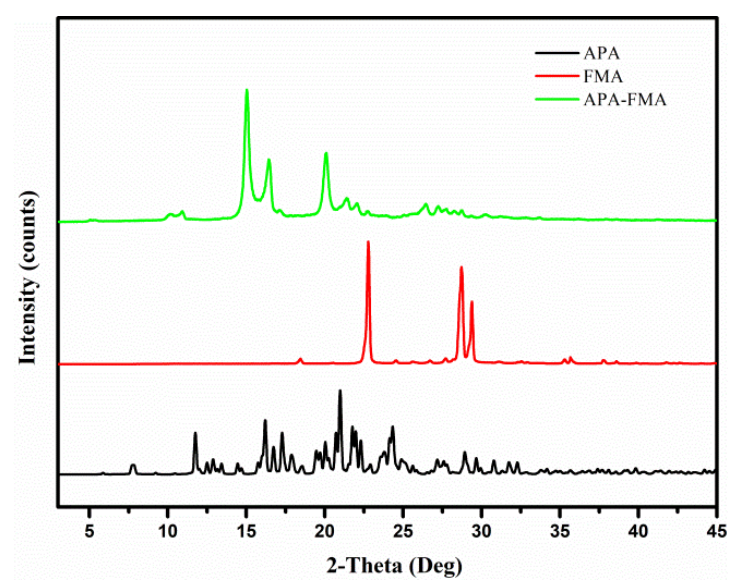

APA-FMA

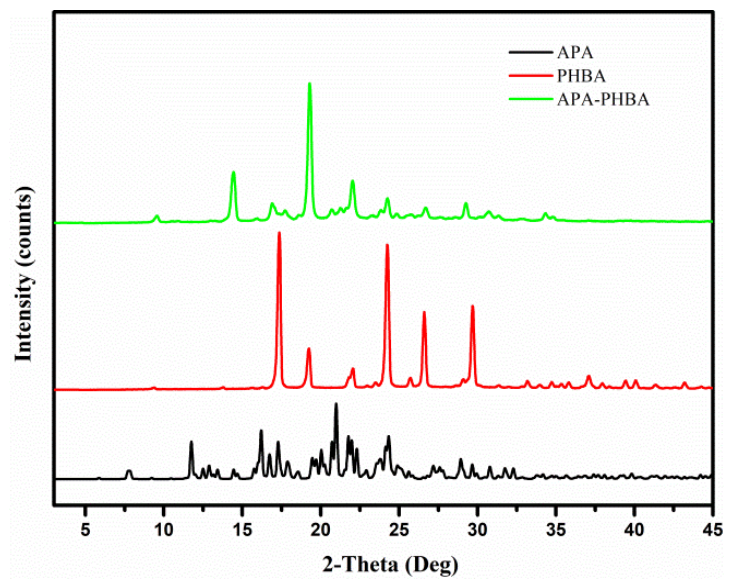

APA-PHBA

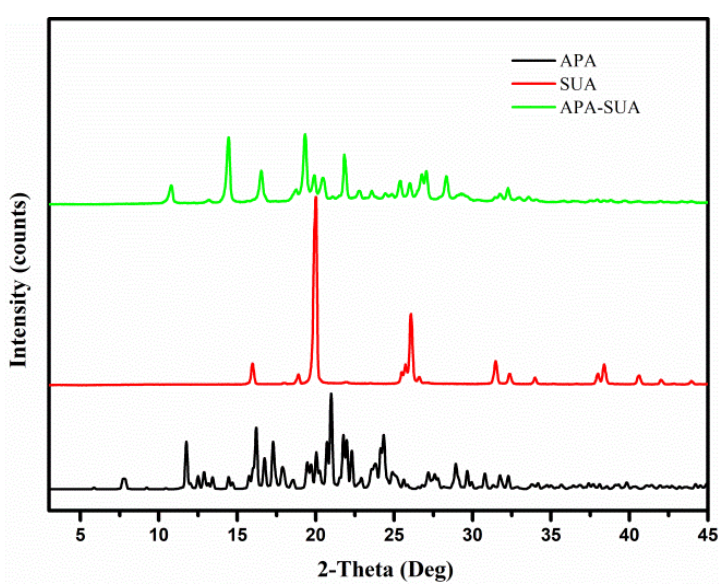

APA-SUA

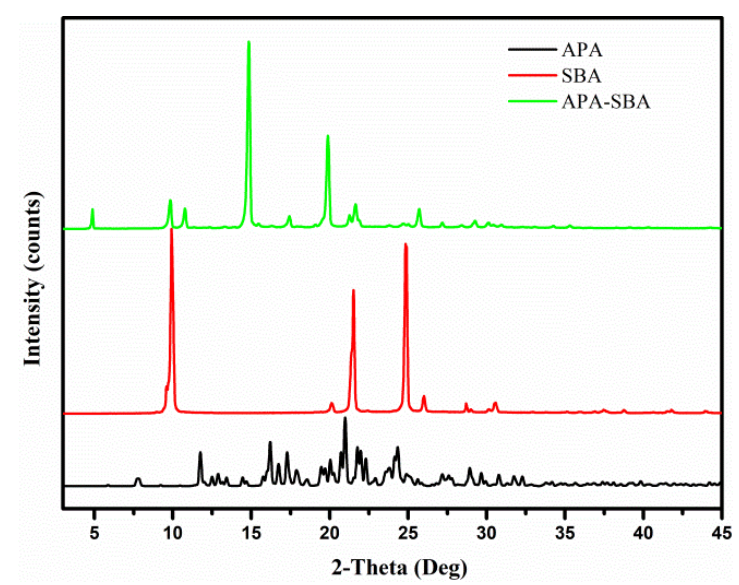

APA-SBA

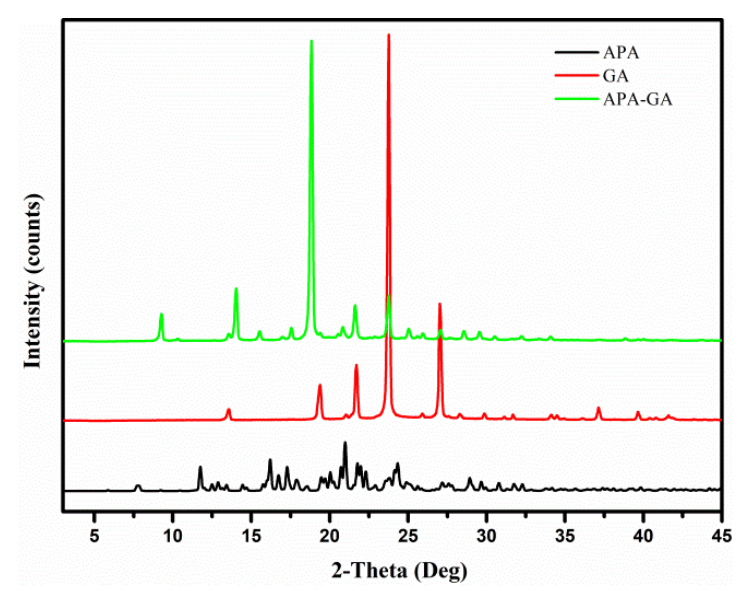

APA-GA 


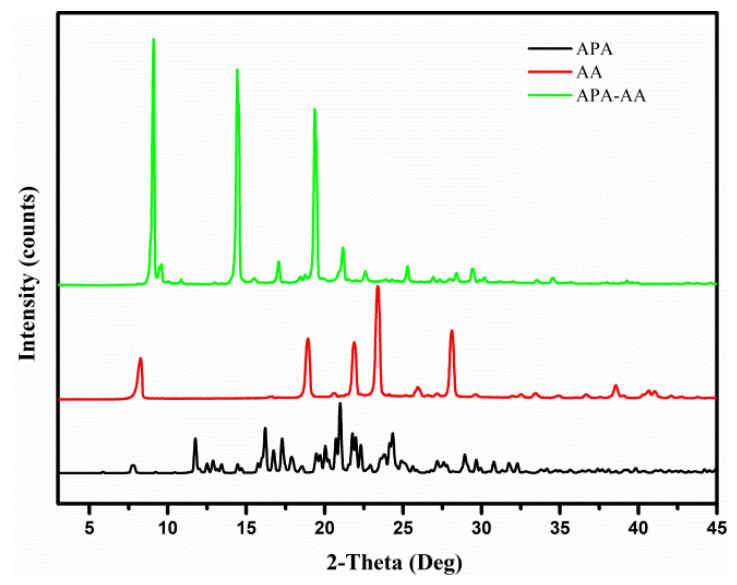

APA-AA

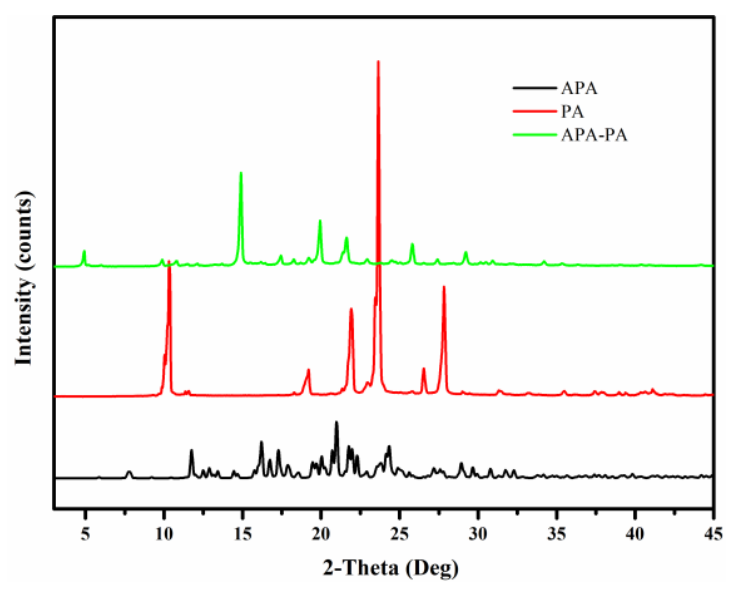

APA-PA

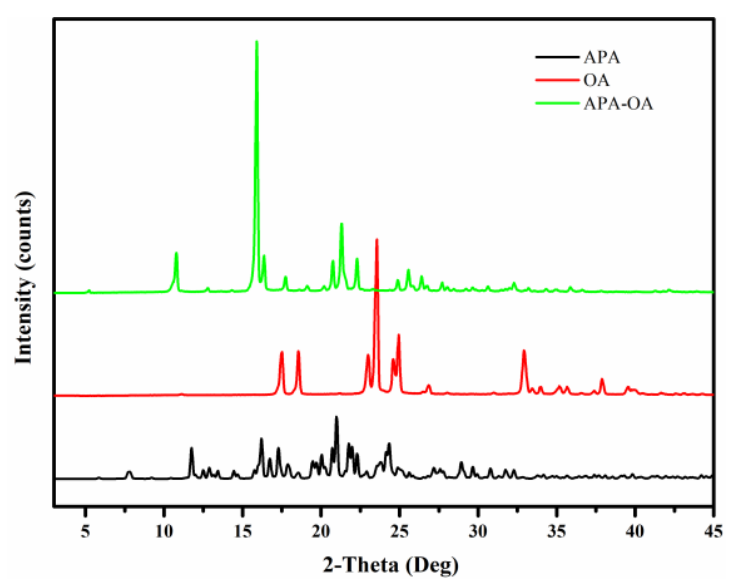

APA-OA

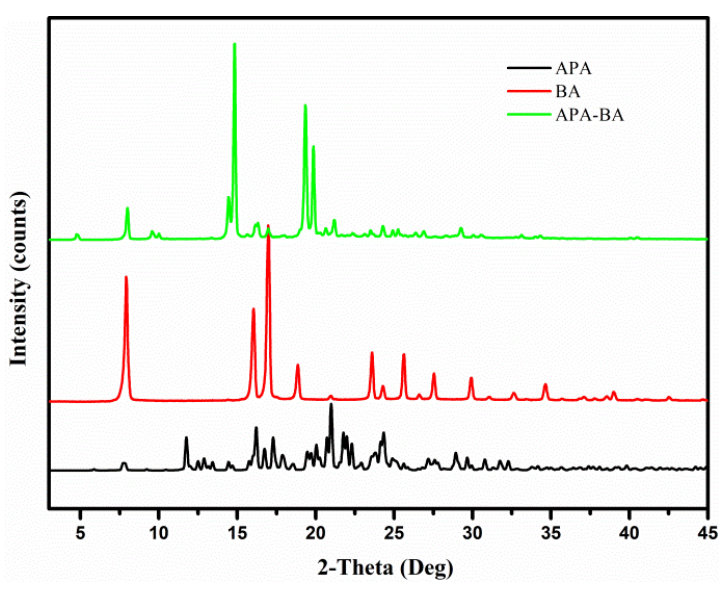

APA-BA

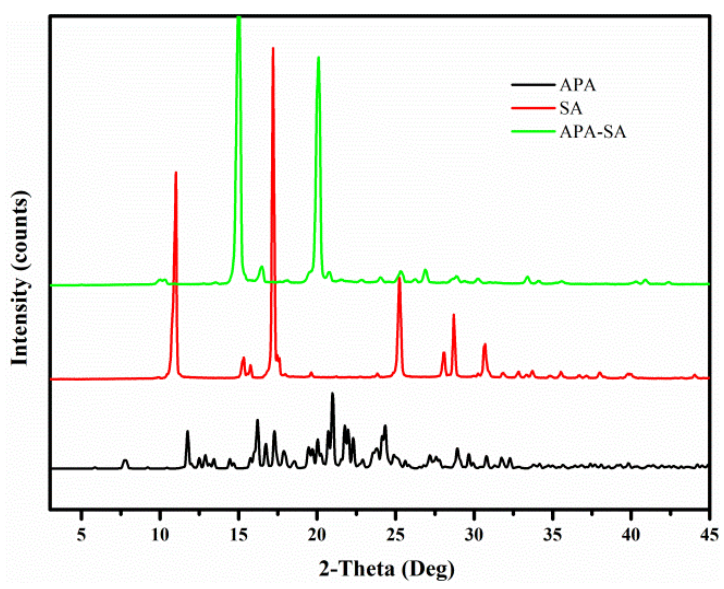

APA-SA

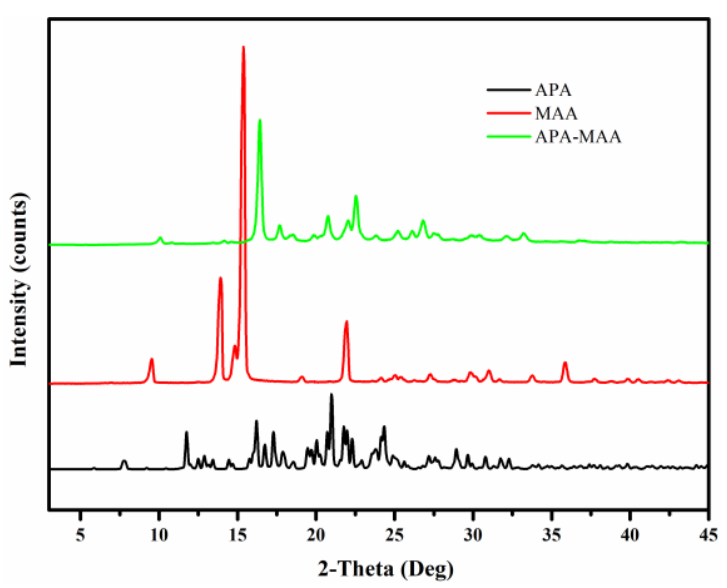

APA-MAA 


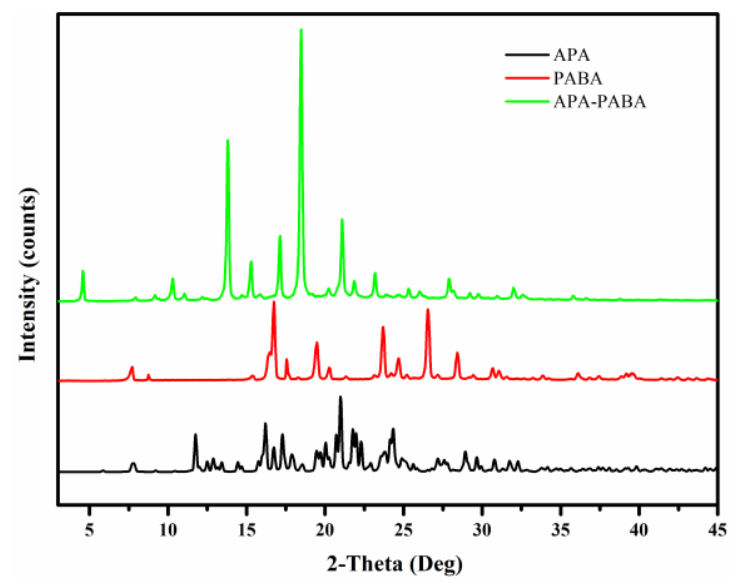

APA-PABA

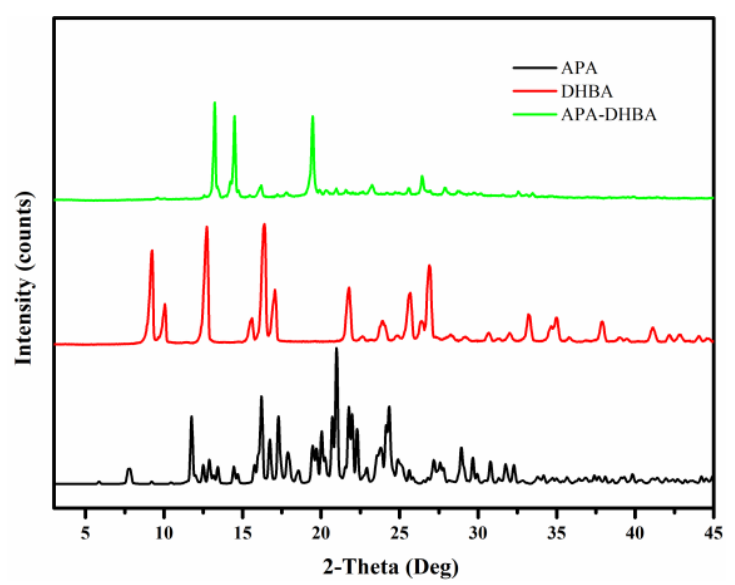

APA-DHBA

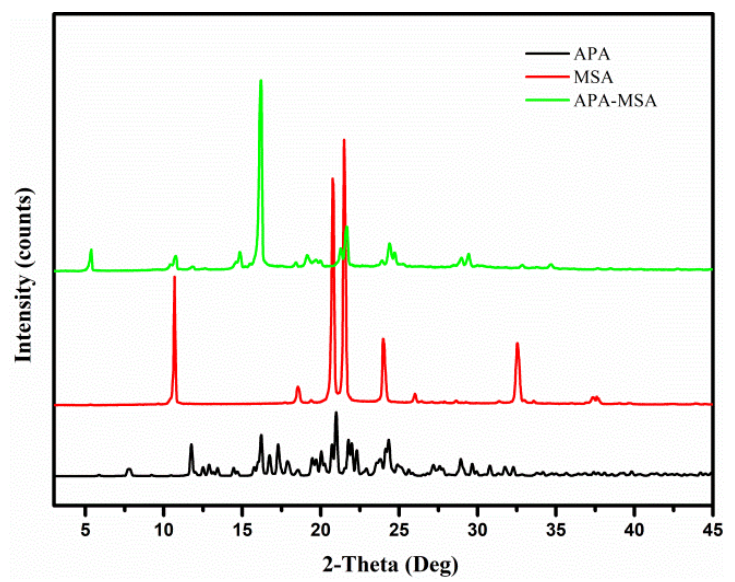

APA-MSA

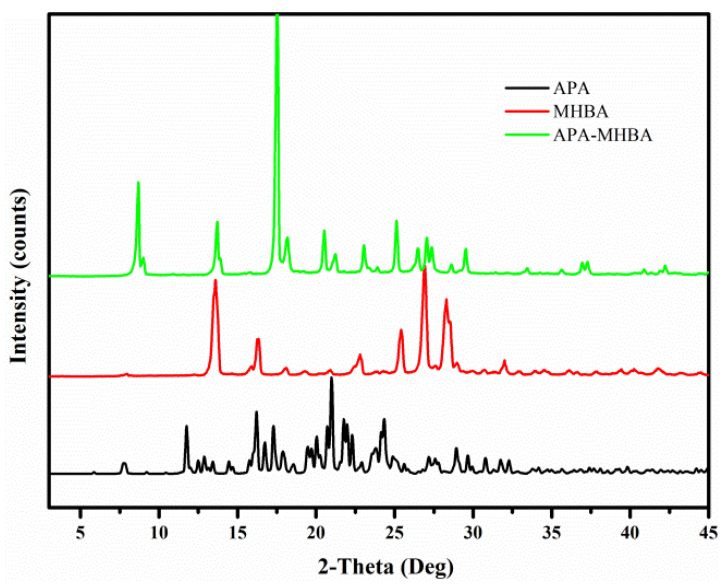

APA-MHBA

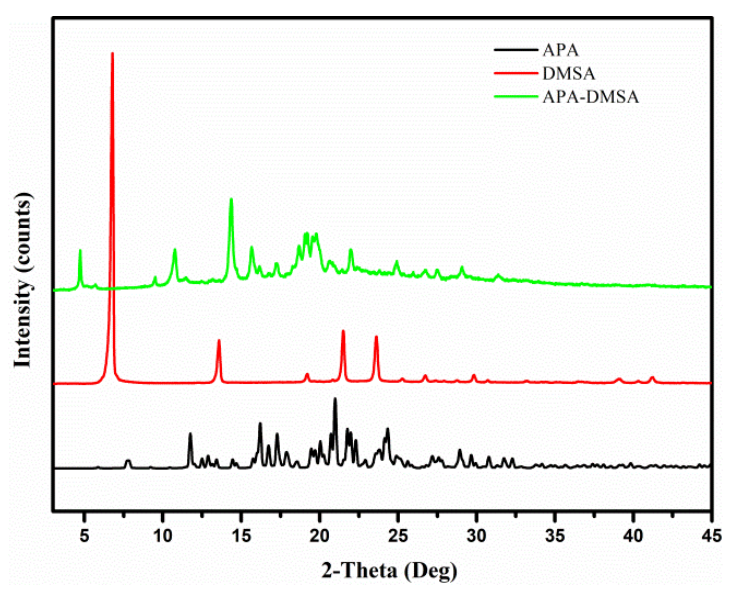

APA-DMSA

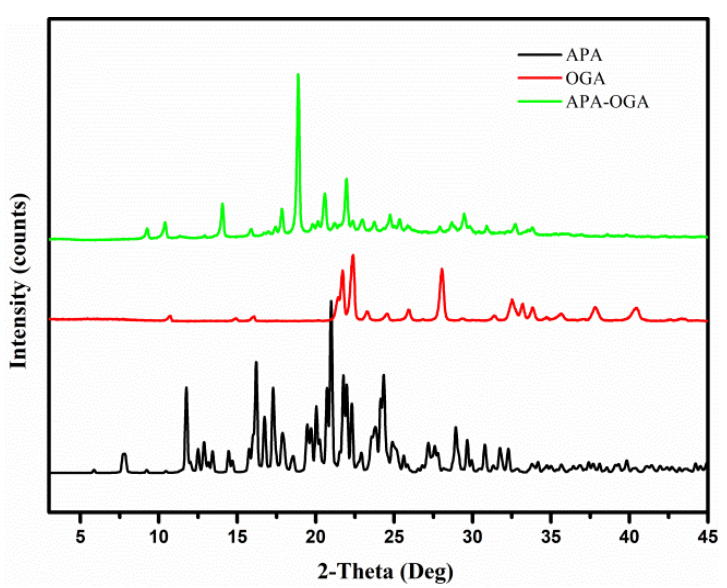

APA-OGA 


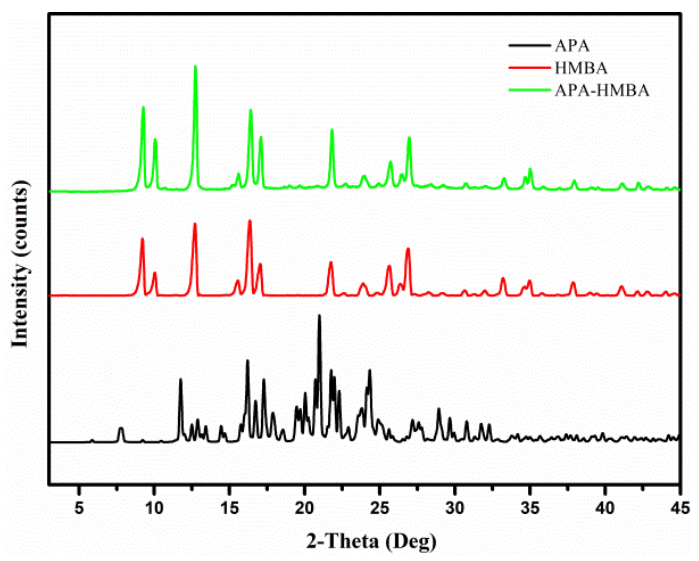

APA-HMBA

Figure S3. PXRD patterns of APA, coformers, and cocrystals.

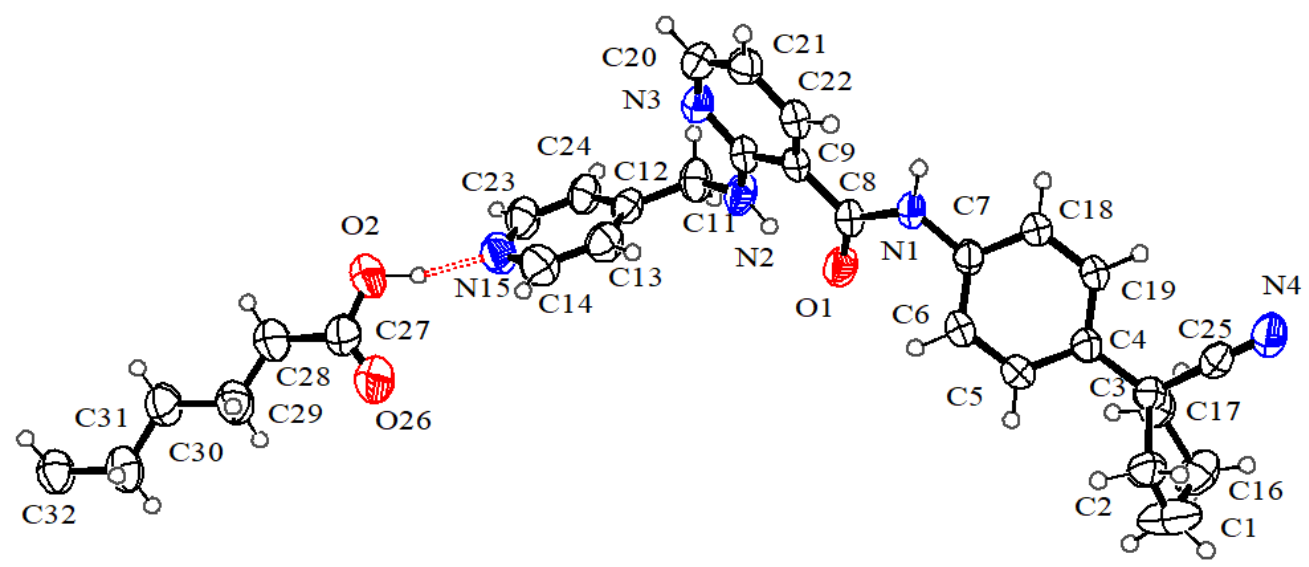

Figure S4. Labelled plots of the asymmetric unit of APA-DDA Cocrystal. 


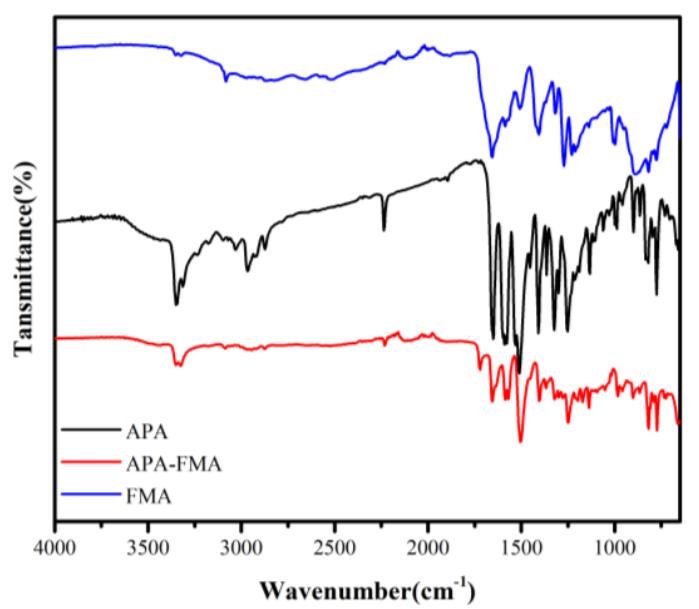

APA-FMA

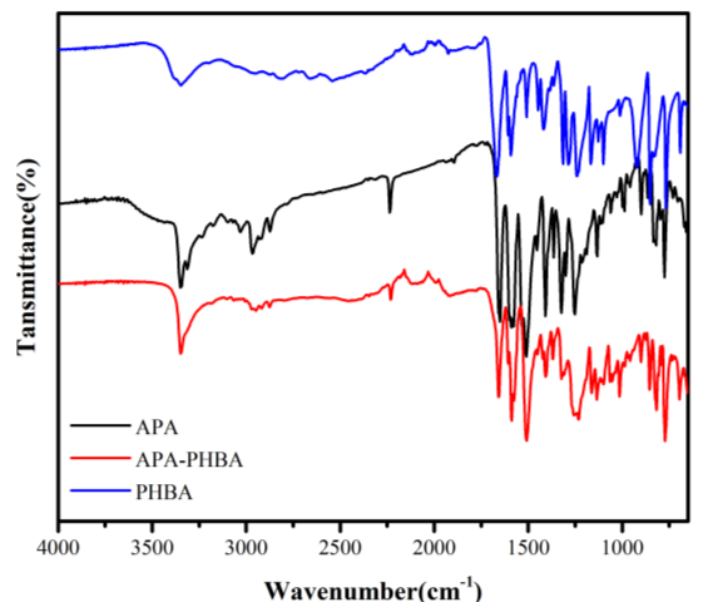

APA-PHBA

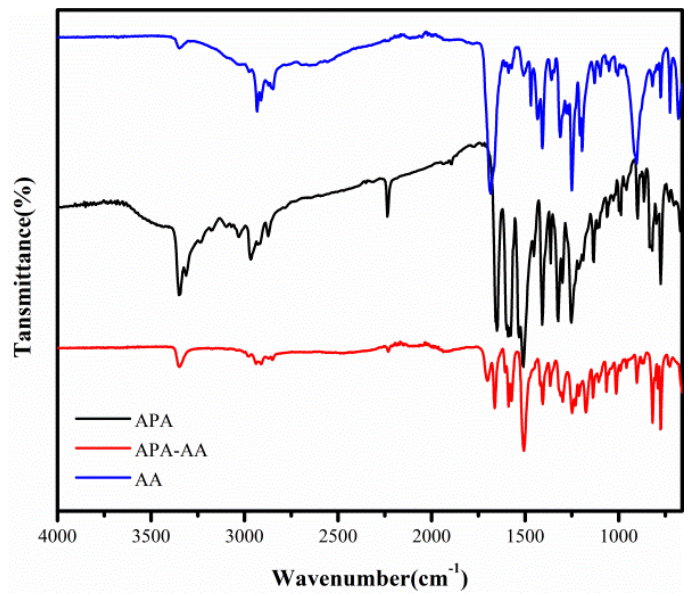

APA-AA

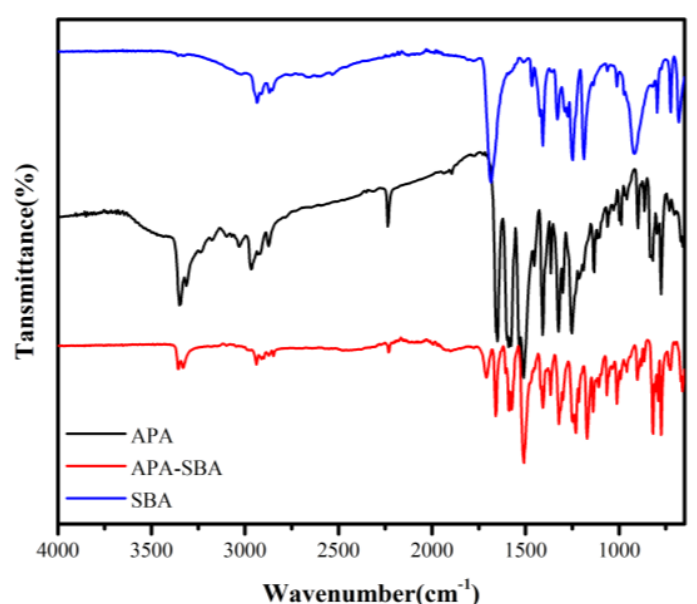

APA-SBA

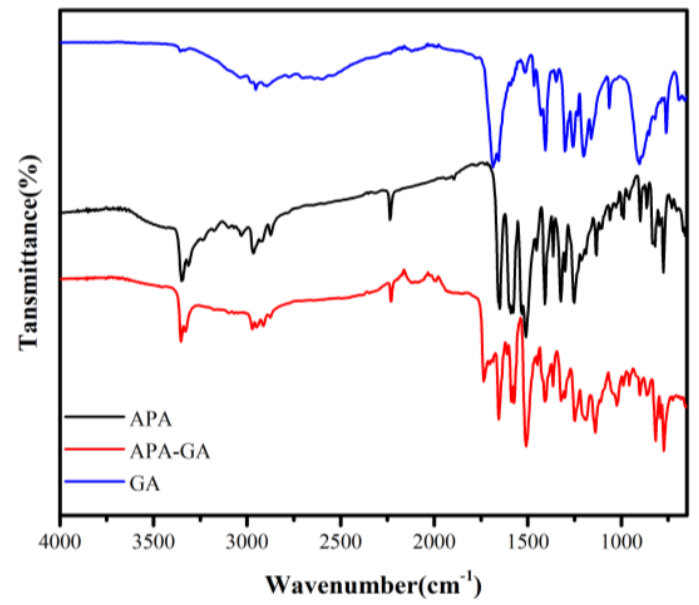

APA-GA

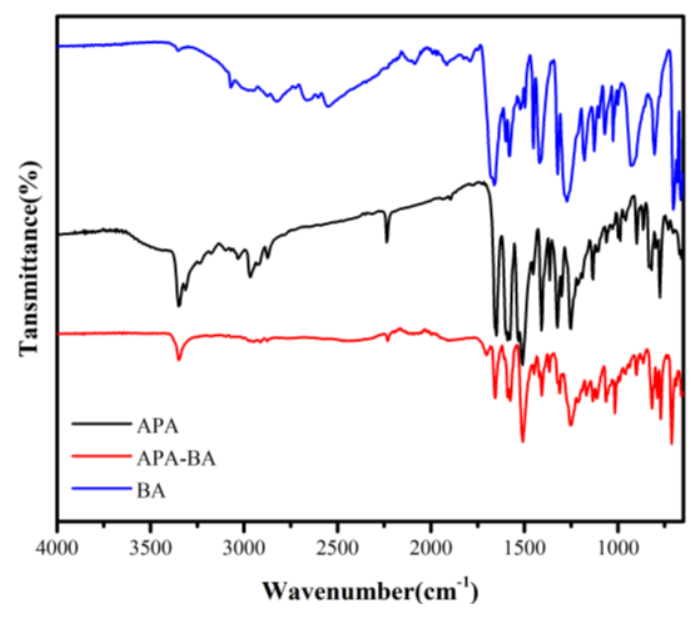

APA-BA 


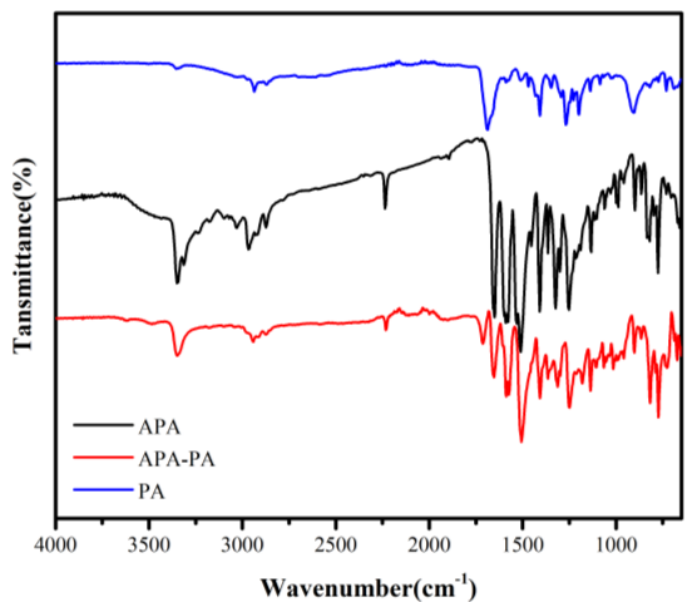

APA-PA

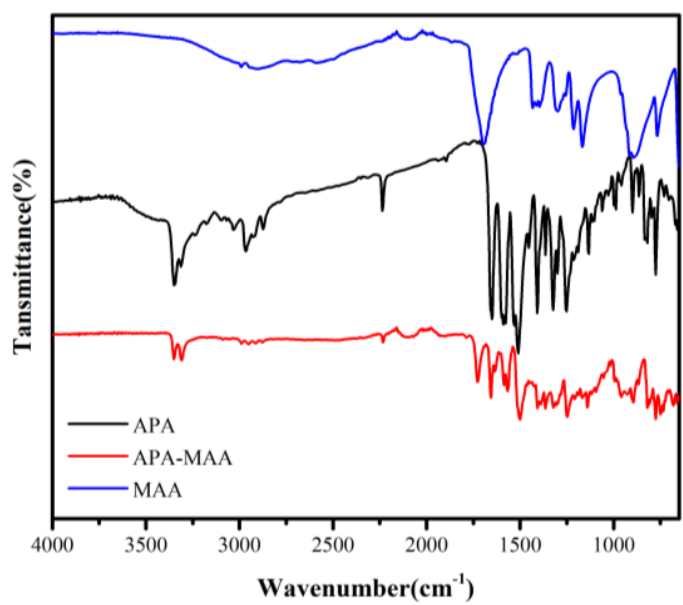

APA-MAA

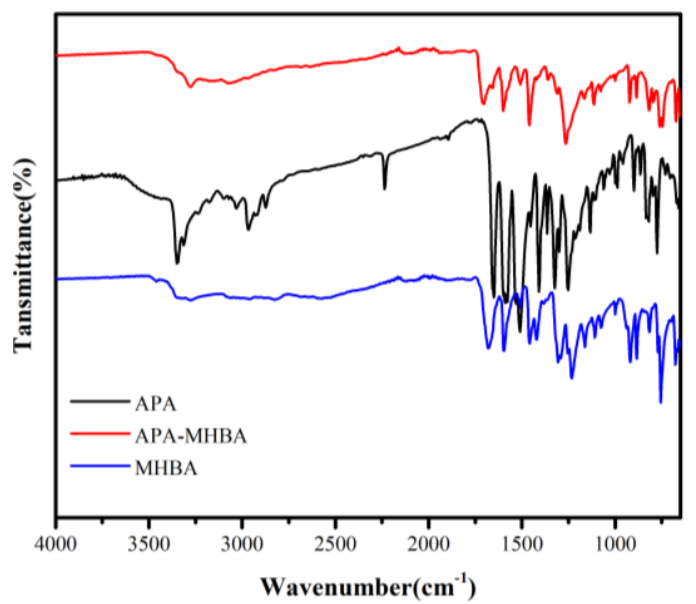

APA-MHBA

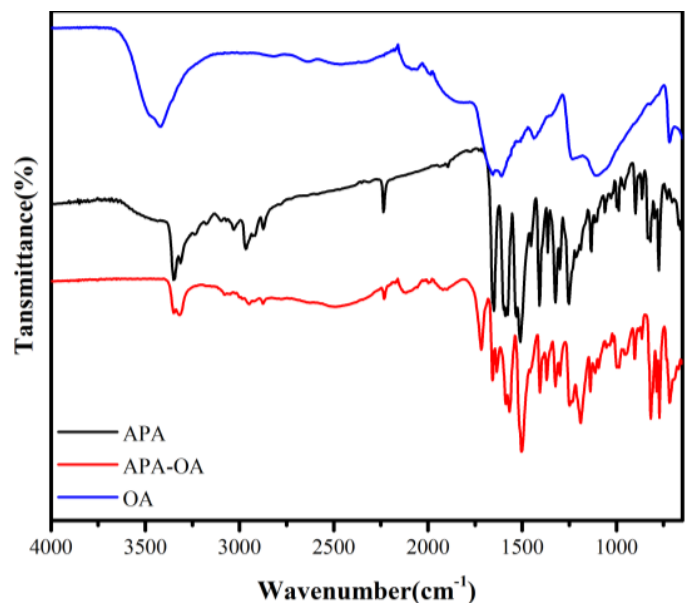

APA-OA

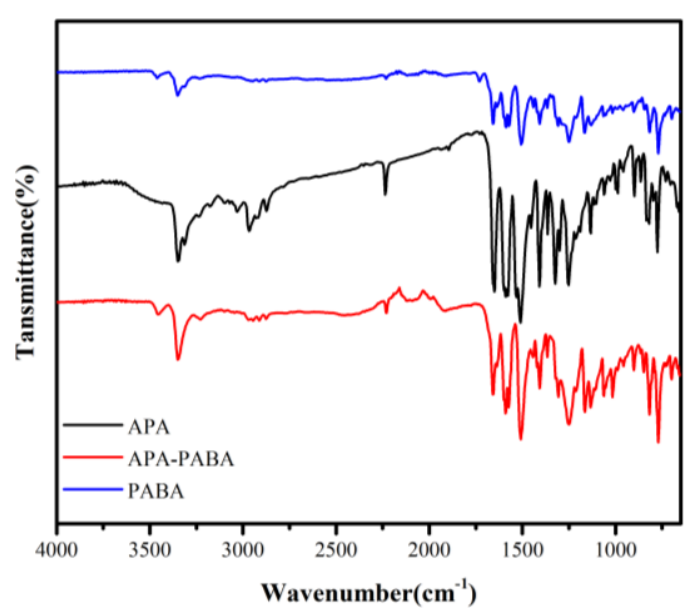

APA-PABA

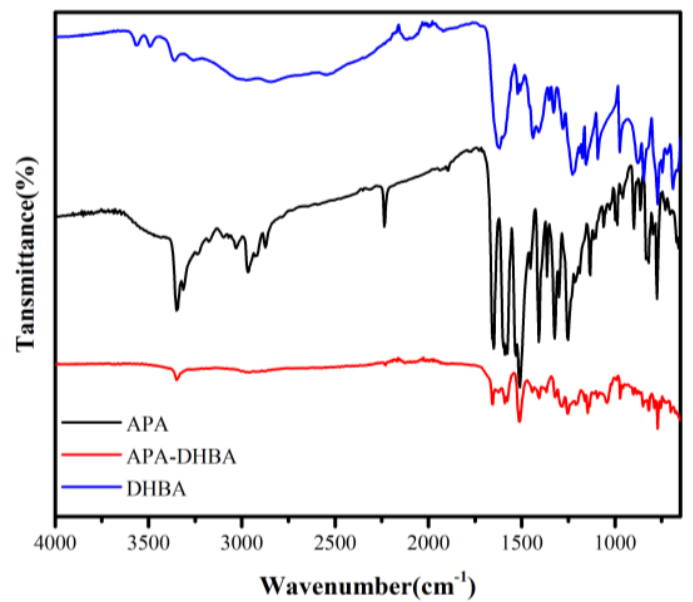

APA-DHBA 


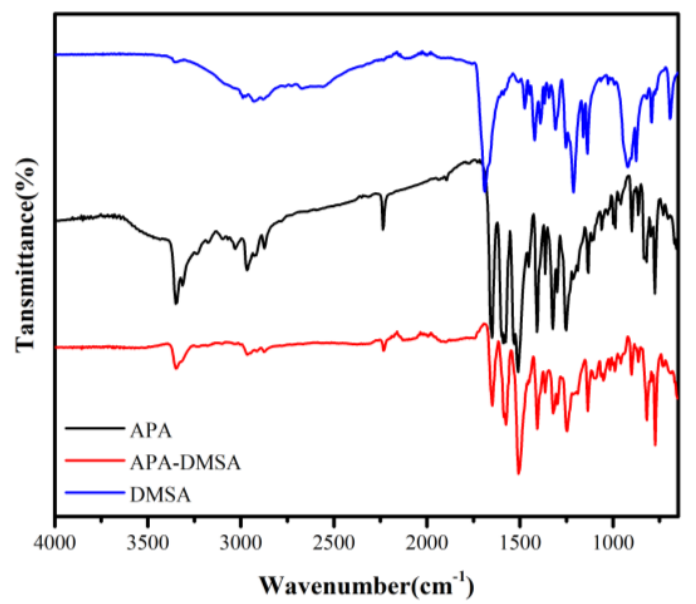

APA-DMSA

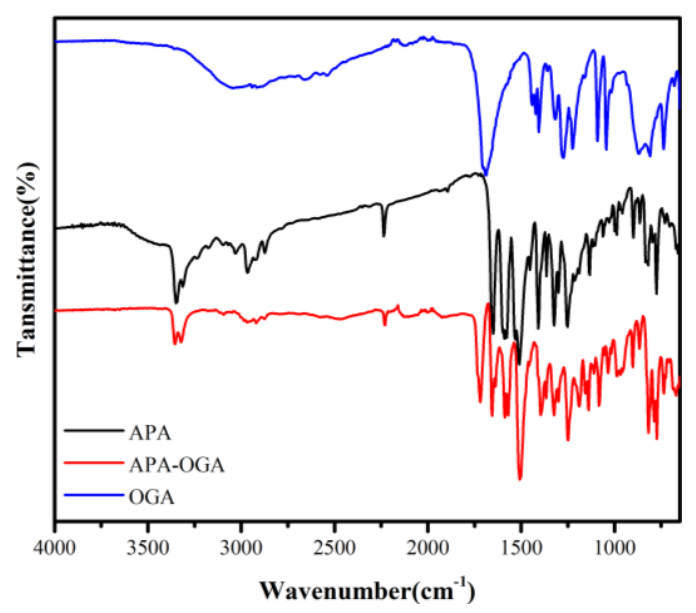

APA-OGA

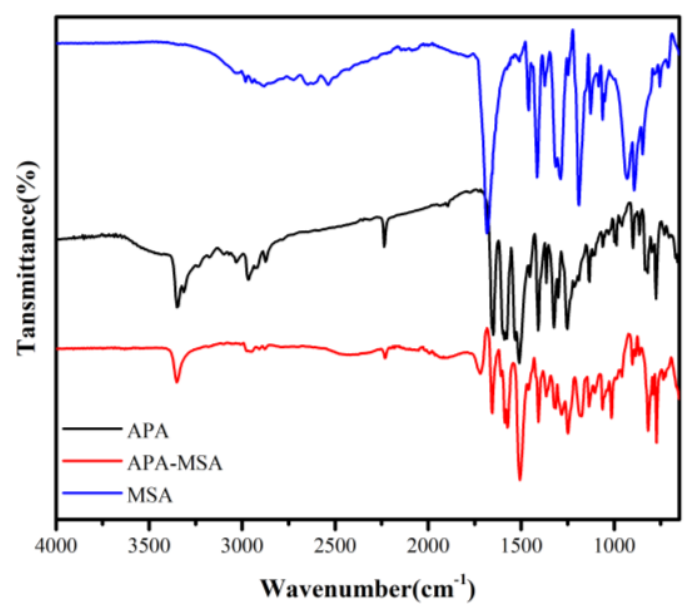

APA-MSA

Figure S5. FT-IR patterns of APA, coformers, and cocrystals. 


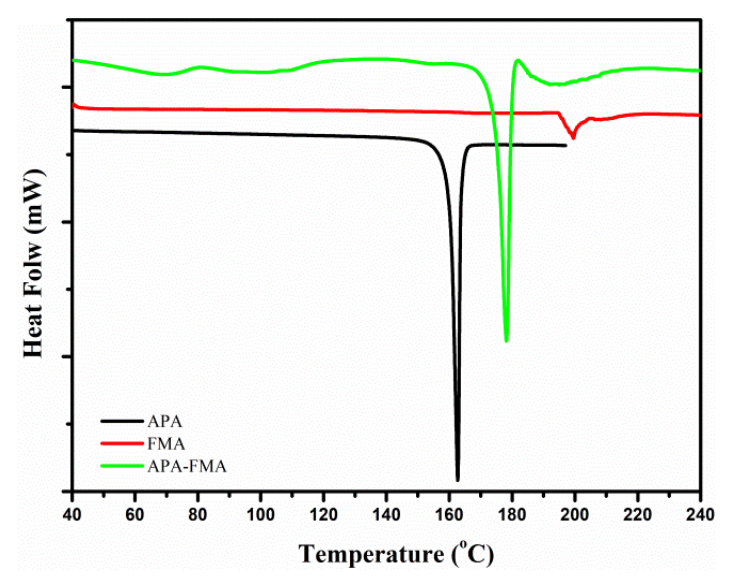

APA-FMA

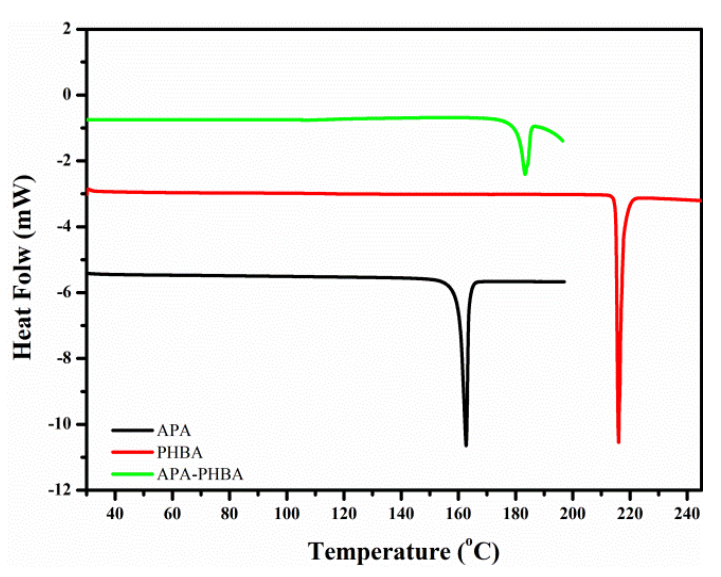

APA-PHBA

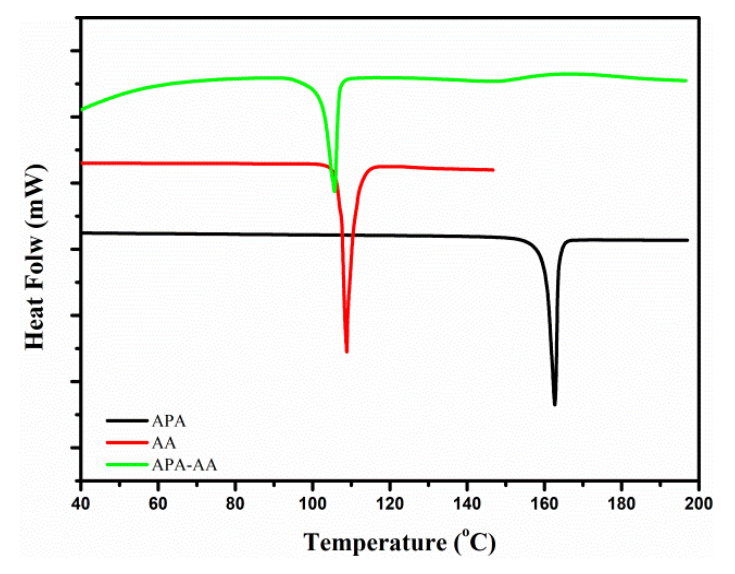

APA-AA

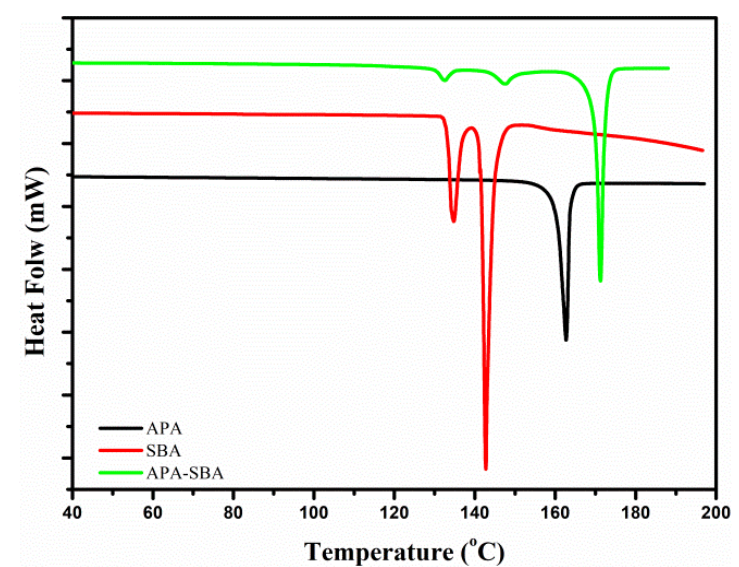

APA-SBA

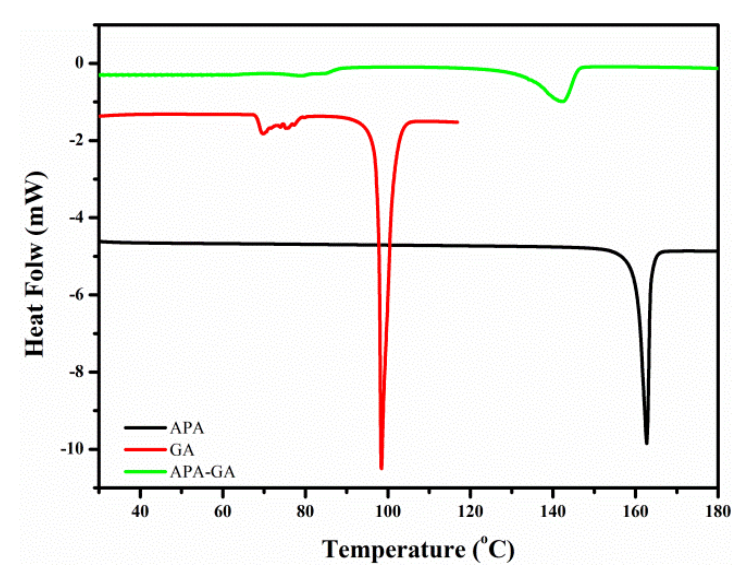

APA-GA

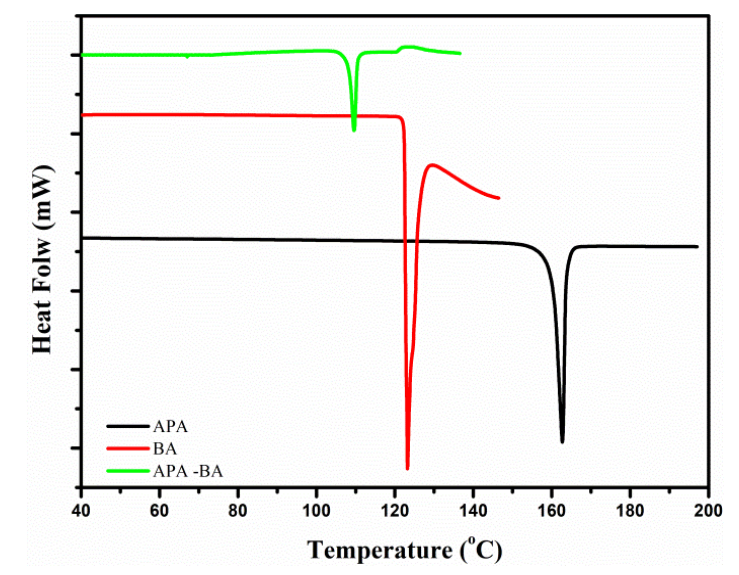

APA-BA 


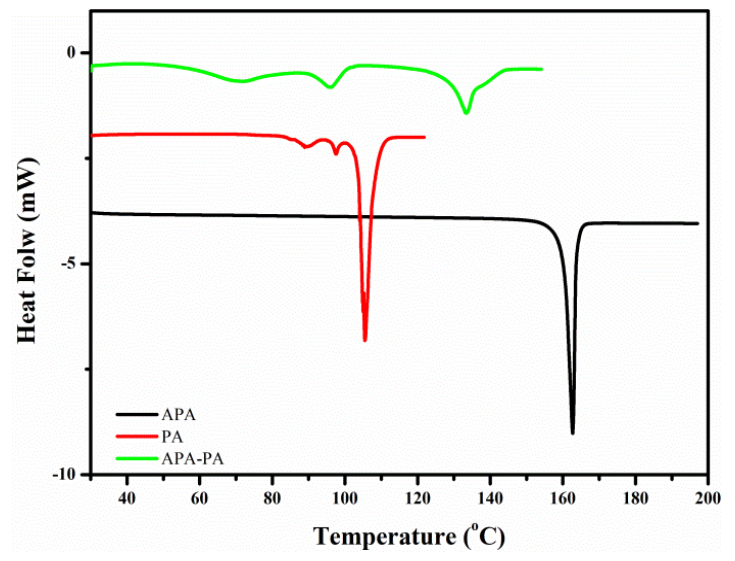

APA-PA

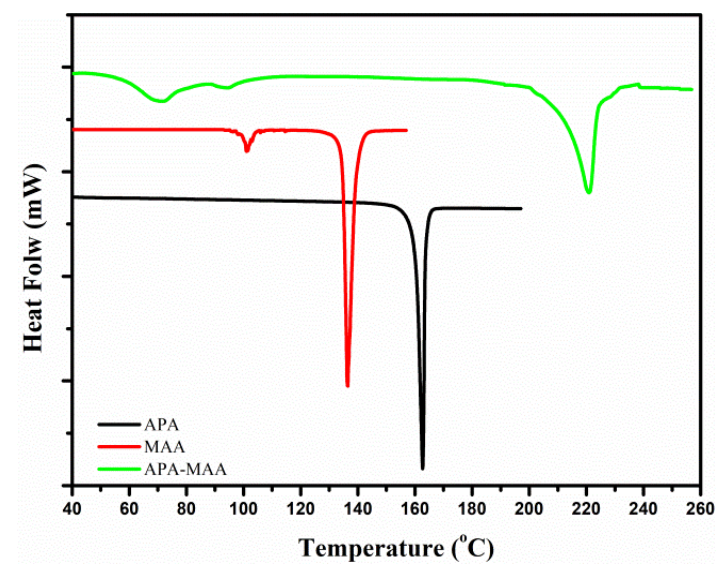

APA-MAA

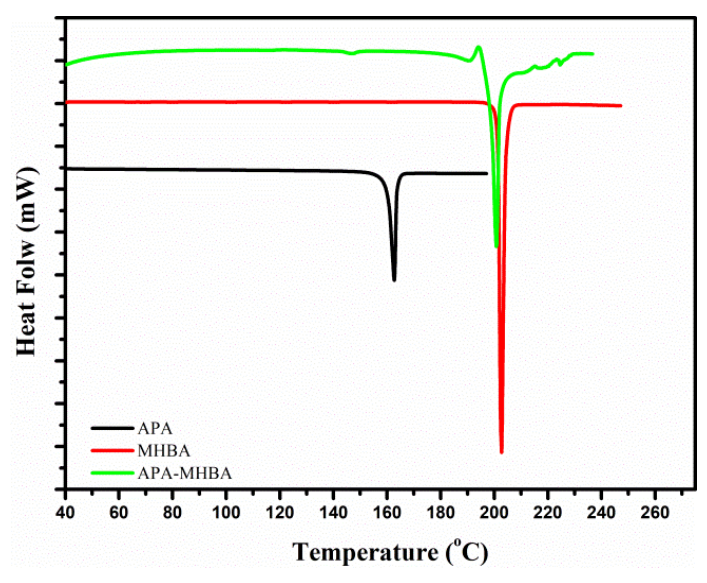

APA-MHBA

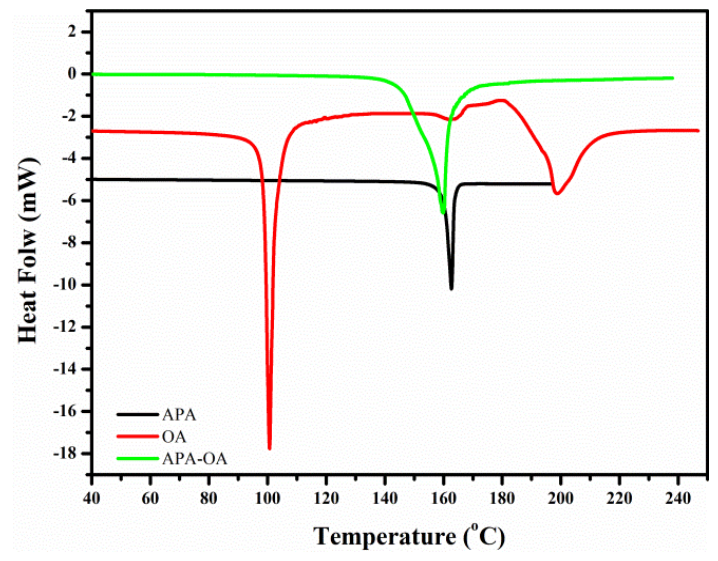

APA-OA

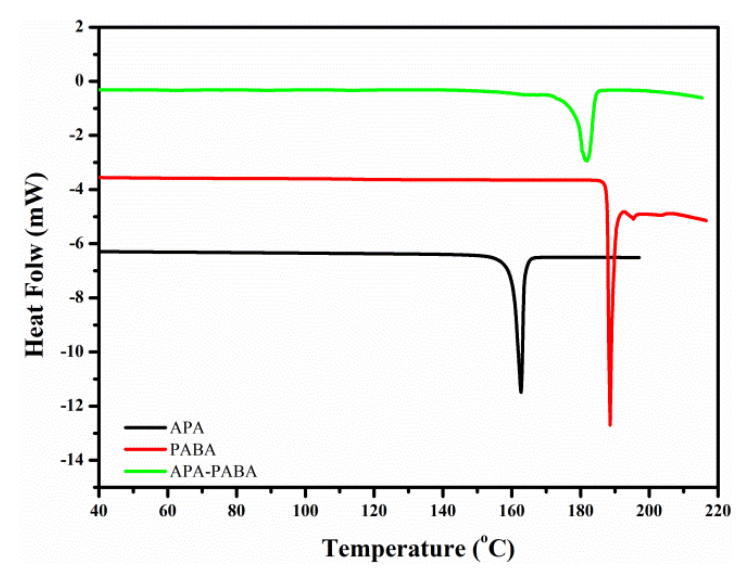

APA-PABA

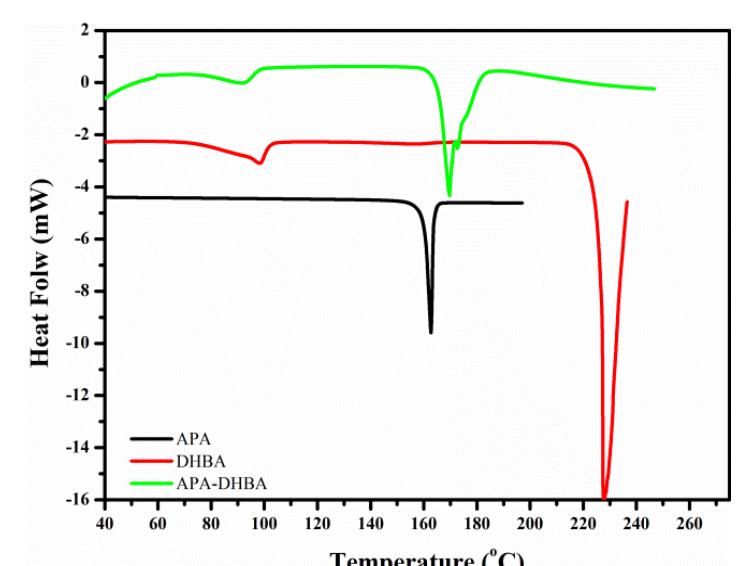

Temperature $\left({ }^{\circ} \mathrm{C}\right)$

APA-DHBA 


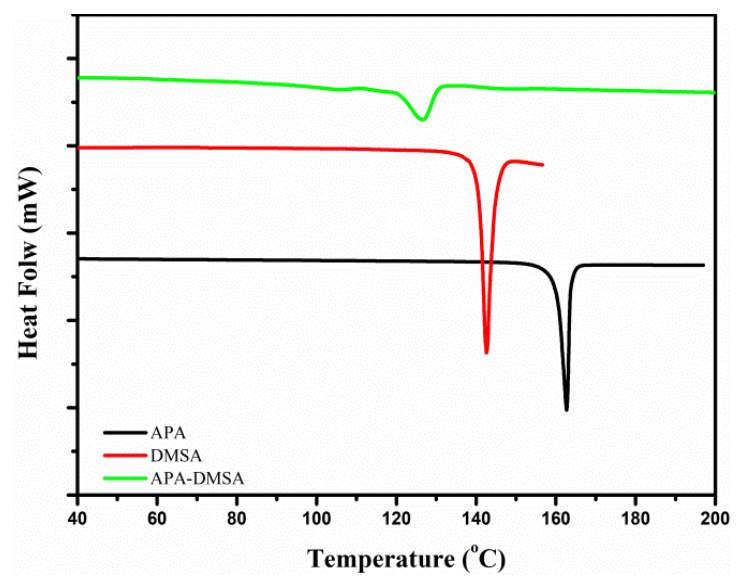

APA-DMSA

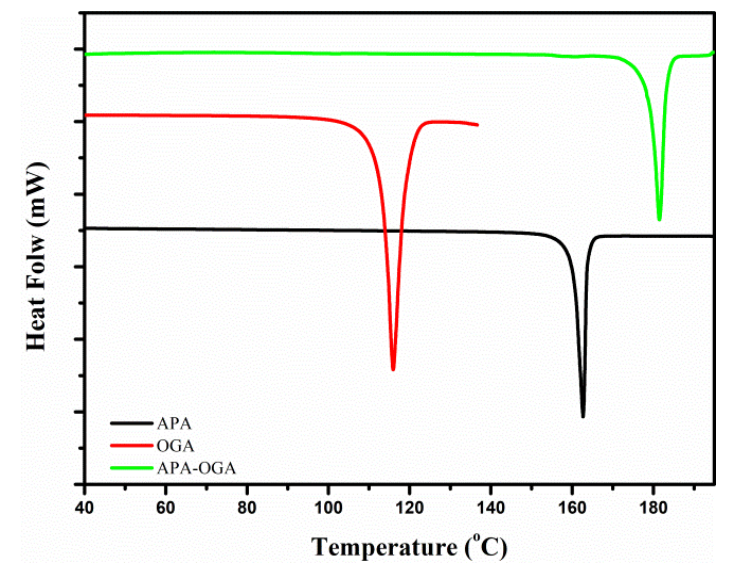

APA-OGA

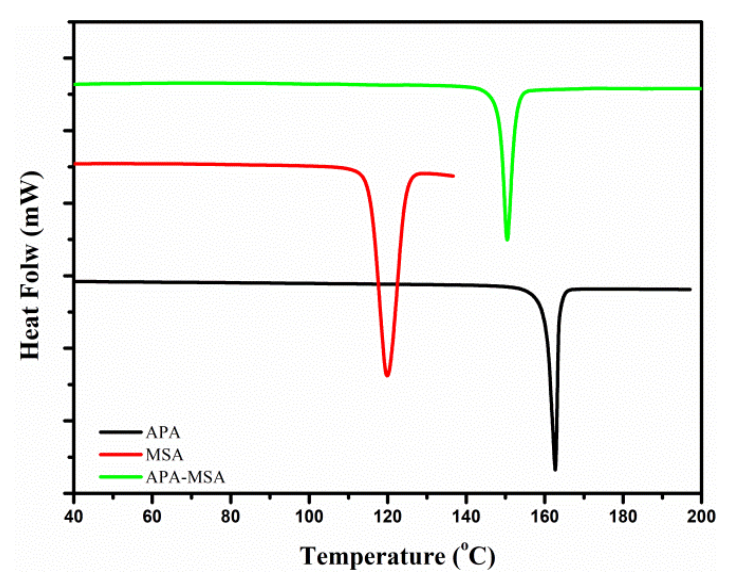

APA-MSA

Figure S6. DSC patterns of APA, coformers, and cocrystals. 

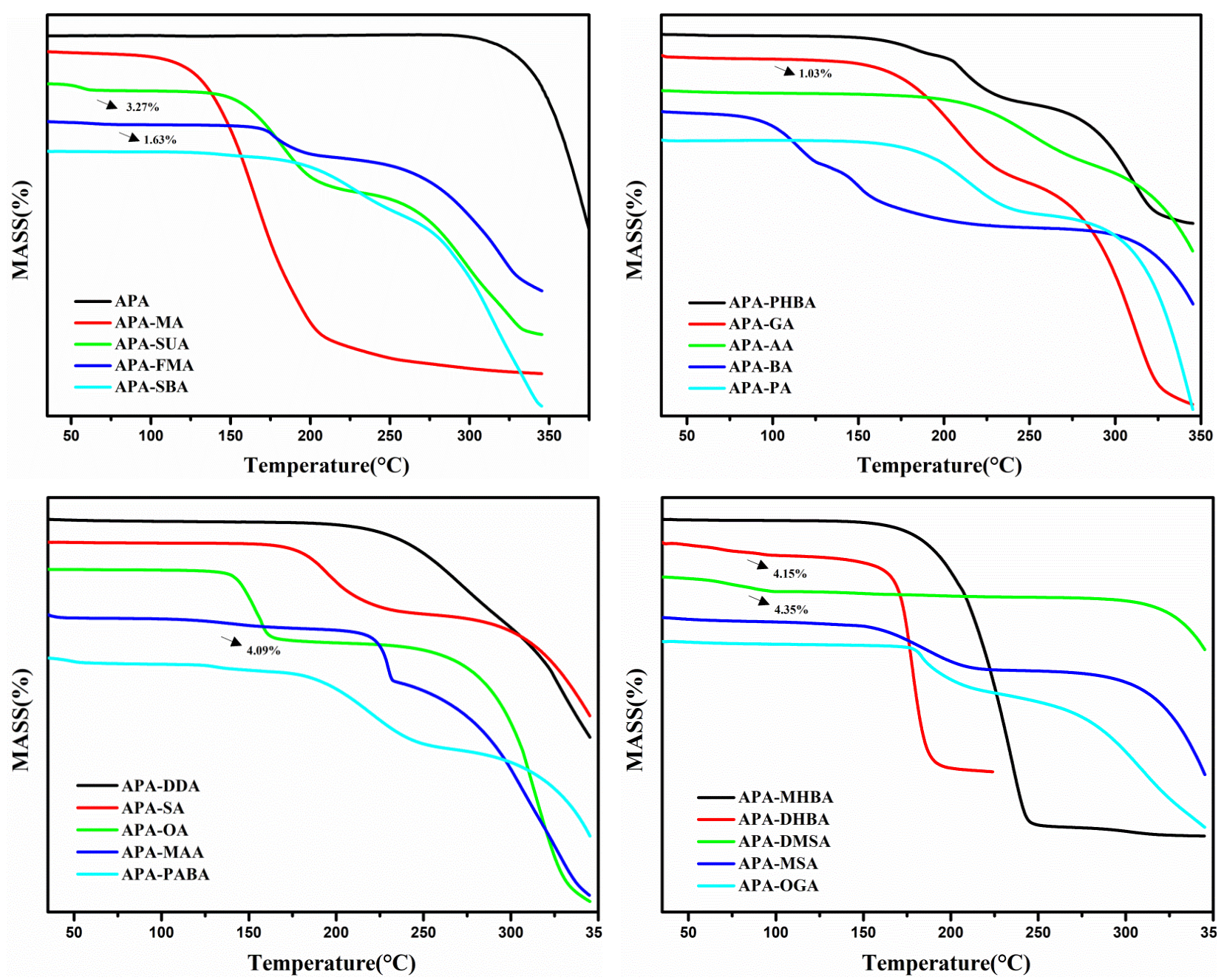

Figure S7. TGA patterns of APA and cocrystals. 
Table S8. The Crystallographic Data of APA-DDA Cocrystal

\begin{tabular}{|c|c|}
\hline Form & APA-DDA \\
\hline Radiation & $\mathrm{CuK} \alpha(\lambda=1.54178)$ \\
\hline Stoichiometry & $2: 1$ \\
\hline Empirical Formula & $\mathrm{C}_{30} \mathrm{H}_{34} \mathrm{~N}_{5} \mathrm{O}_{3}$ \\
\hline Formula weight & 512.62 \\
\hline Temperature/K & $296(2)$ \\
\hline Crystal system & triclinic \\
\hline Space group & $P-1$ \\
\hline$a / \AA$ & $8.8371(10)$ \\
\hline$b / \AA$ & $9.1570(10)$ \\
\hline$c / \AA$ & $19.406(2)$ \\
\hline$\alpha /^{\circ}$ & $88.138(6)$ \\
\hline$\beta /^{\circ}$ & $88.520(6)$ \\
\hline$\gamma /{ }^{\circ}$ & $61.529(4)$ \\
\hline Cell volume $/ \AA^{3}$ & $1379.6(3)$ \\
\hline $\mathrm{Z}$ & 2 \\
\hline$\rho_{\text {calc }} / \mathrm{g} \cdot \mathrm{cm}^{-3}$ & 1.234 \\
\hline Independent reflns & 4697 \\
\hline$R_{\text {int }}$ & 0.0655 \\
\hline$R_{\text {sigma }}$ & 0.0331 \\
\hline S & 1.066 \\
\hline$R_{1}$ & 0.0551 \\
\hline$w R_{2}$ & 0.1448 \\
\hline
\end{tabular}


Table S9. The Hydrogen Bonding Details of APA-DDA Cocrystal

\begin{tabular}{|c|c|c|c|c|c|}
\hline $\mathrm{Nr}$ & Donor-H $\cdots$ Acceptor & $\mathrm{D}-\mathrm{H} / \AA$ & $\mathrm{H} \cdots \mathrm{A} / \AA$ & $\mathrm{D} \cdots \mathrm{A} / \AA$ & $\mathrm{D}-\mathrm{H} \cdots \mathrm{A} /{ }^{\circ}$ \\
\hline 1 & $\mathrm{~N}(1)-\mathrm{H}(1) \cdots \mathrm{N}(4)$ & $0.90(2)$ & $2.30(3)$ & $3.159(3)$ & $160(2)$ \\
\hline 2 & $\mathrm{~N}(2)-\mathrm{H}(2) \cdots \mathrm{O}(1)$ & $0.88(3)$ & $2.00(3)$ & $2.694(3)$ & $134(2)$ \\
\hline 3 & $\mathrm{O}(2)-\mathrm{H}(2 \mathrm{C}) \cdots \mathrm{N}(15)$ & $1.02(5)$ & $1.69(5)$ & $2.686(4)$ & $164(4)$ \\
\hline 4 & $\mathrm{C}(6)-\mathrm{H}(6) \cdots \mathrm{O}(1)$ & 0.93 & 2.47 & $2.886(3)$ & 107 \\
\hline 5 & $\mathrm{C}(13)-\mathrm{H}(13) \cdots \mathrm{N}(2)$ & 0.93 & 2.6 & $2.914(4)$ & 101 \\
\hline 6 & $\mathrm{C}(17)-\mathrm{H}(17 \mathrm{~B}) \cdots \mathrm{O}(1)$ & 0.97 & 2.46 & $3.398(3)$ & 162 \\
\hline
\end{tabular}




\section{Comparison with Previous Methods}

Table S10. Experimental Screening Results and HBP, HBC, HBE, and LRW Prediction Results for Coformers Targeting Nevirapine ${ }^{\text {a }}$

\begin{tabular}{|c|c|c|c|c|c|}
\hline & Exp. & HBP & $\mathrm{HBC}$ & HBE & LRW \\
\hline Oxalic acid & Yes & Yes & Yes & Yes & Yes \\
\hline Glutaric acid & Yes & Yes & Yes & Yes & Yes \\
\hline Citric acid & Yes & Yes & Yes & Yes & Yes \\
\hline Gallic acid & Yes & Yes & Yes & Yes & Yes \\
\hline Ferulic acid & Yes & Yes & Yes & Yes & No \\
\hline Tartaric acid & Yes & Yes & Yes & Yes & No \\
\hline Urea & Yes & No & No & Yes & Yes \\
\hline Mandelic acid & Yes & Yes & Yes & Yes & No \\
\hline Cinnamic acid & Yes & Yes & Yes & No & Yes \\
\hline Hippuric acid & Yes & Yes & Yes & Yes & No \\
\hline Benzoic acid & Yes & Yes & Yes & Yes & Yes \\
\hline 4-hydroxybenzoic acid & Yes & Yes & Yes & Yes & Yes \\
\hline Malonic aid & Yes & Yes & Yes & Yes & Yes \\
\hline Salicylic acid & Yes & N.A. & N.A. & N.A. & Yes \\
\hline Saccharin & Yes & N.A. & N.A. & N.A. & Yes \\
\hline \multicolumn{6}{|c|}{$\begin{array}{l}\text { a Items are shown in bold if predictions accord with experimental results. Coformers at top } 10 \% \\
\text { of the recommendation list were viewed as positive predictions by LRW. Experimental and } \\
\text { predictive positives are labeled as "Yes", and experimental and predictive negatives are labeled } \\
\text { as "No". Unobtainable data are labeled as "N.A.". Nevirapine-maleic acid was existing in our } \\
\text { cocrystal network and was omitted. }\end{array}$} \\
\hline
\end{tabular}


Table S11. Experimental Screening Results and HBP, HBC, HBE, and LRW Prediction Results for Coformers Targeting Diclofenac ${ }^{\text {a }}$

\begin{tabular}{|c|c|c|c|c|c|}
\hline & Exp. & HBP & $\mathrm{HBC}$ & HBE & LRW \\
\hline 2-aminopyridine & Salt & Yes & Yes & Yes & No \\
\hline 3-aminopyridine & Salt & No & Yes & Yes & Yes \\
\hline 2-amino-5-chloropyridine & Yes & Yes & Yes & Yes & Yes \\
\hline 2-amino-3,5-dibromopyridine & Yes & Yes & Yes & Yes & No \\
\hline 3-hydroxypyridine & Yes & Yes & Yes & No & No \\
\hline 2-amino-4-hydroxy-6-methylpyrimidine & Yes & Yes & No & Yes & No \\
\hline 2-aminopyrimidine & Yes & No & Yes & Yes & Yes \\
\hline 2-amino-4,6-dimethylpyrimidine & Yes & Yes & Yes & Yes & Yes \\
\hline 2-amino-4-chloro-6-methylpyrimidine & Yes & Yes & Yes & No & Yes \\
\hline 4-Chloro-2,6-diaminopyrimidine & Yes & No & Yes & No & No \\
\hline 3,5-dimethylpyrazole & No & No & Yes & Yes & Yes \\
\hline Pyrazole & No & No & Yes & Yes & No \\
\hline 3,5-dimethyl-4-chloropyrazole & No & No & Yes & No & Yes \\
\hline 4-iodo-3,5-dimethylprazole & No & No & Yes & Yes & No \\
\hline 4-Bromopyrazole & No & No & Yes & Yes & No \\
\hline 4-Iodopyrazole & No & No & Yes & Yes & No \\
\hline 4,4'-bipyridine & Yes & N.A. & N.A. & N.A. & Yes \\
\hline theophylline & Yes & N.A. & N.A. & N.A. & Yes \\
\hline
\end{tabular}

\footnotetext{
${ }^{a}$ Items are shown in bold if predictions accord with experimental results. Coformers at top $10 \%$ of the recommendation list were viewed as positive predictions by LRW. Experimental and predictive positives are labeled as "Yes", and experimental and predictive negatives are labeled as "No". Unobtainable data are labeled as "N.A.".
} 


\section{References}

(1) Francart, T.; Wieringen, A. v.; Wouters, J., APEX 3: a multi-purpose test platform for auditory psychophysical experiments. Journal of Neuroscience Methods 172, 283-293.

(2) SAINT, APEX2, and APEX3 Software for CCD Diffractometers. ed.; 2014.

(3) Sheldrick, G. M., SADABS Multi Scan Absorption. 2016.

(4) Sheldrick, G., SAINT and XPREP, Siemens Industrial Automation Inc. Madison, WI. 1995.

(5) Sheldrick, G. M., Crystal structure refinement with SHELXL. Acta Crystallogr., Sect. C: Struct. Chem. 2015, 71, 3-8.

(6) Sheldrick, G. M.; IUCr, A short history of SHELX. 2008, 64, 112-122.

(7) Dolomanov, O. V., A Bourhis, Luc J., A Gildea, Richard J., A Howard, Judith A. K., A Puschmann, Horst, OLEX2: a complete structure solution, refinement and analysis program. Journal of Applied Crystallography 2009, 42. 